REVISTA DE DERECHO UNED, NÚM. 12, 2013

\title{
LE IMPUGNAZIONI INCIDENTALI NEL PROCESSO AMMINISTRATIVO ITALIANO
}

\author{
THE INSTITUTION OF THE INCIDENTAL APPEAL OF THE \\ CODE OF CIVIL PROCEDURE
}

\begin{abstract}
CARMENCITA GuACCI
Ricercatrice a.C. Di diritto amministrativo e professoressa aggregata di diritto amministrativo Università Pegaso
\end{abstract}

Sintesi: L'istituto dell'impugnazione incidentale costituisce applicazione del principio di concentrazione delle impugnazioni. L'art. 96 c.p.a. disciplina le impugnazioni incidentali, ammettendole per l'appello, per la revocazione, per l'opposizione di terzo e, con qualche particolarità, per il ricorso per cassazione. L'impugnazione va proposta in forma incidentale, a pena di decadenza, dai destinatari della notifica della sentenza e dell'atto di integrazione del contraddittorio. Il rapporto di condizionalità-dipendenza, fra impugnazione principale e impugnazione incidentale, sussiste sia nel caso di impugnazione incidentale propria, sia nel caso di impugnazione incidentale tardiva. L'esigenza di applicare in maniera rigorosa il principio di concentrazione delle impugnazioni ha indotto il legislatore a sancire l'ammissibilità dell'impugnazione incidentale anche avverso capi autonomi della sentenza. tivo.

Parole chiave: impugnazione incidentale, processo amministra-

Abstract: The institution of the incidental appeal corresponds with the application of the principle of the appeals concentration.

The article number 96 of the code of civil procedure governs the incidental objections, accepting them to the judicial review, the new 
trial, the examination pro interesse suo, and, in certain events, the petition to the Highest Court.

The appeal has to be proposed by the service of the process adjudication and cross-examination receivers under the vest of an incidental form, otherwise it will run the risk of lapse.

The relationship of conditioning-dependence between the main and the incidental appeals can be considered either in the case of a proper than of an untimely incidental appeal.

The need to strictly apply the principle of the appeals concentration has oriented the legislator to decree the receivability of the incidental appeal also in case of autonomous judgement charges.

Keywords: incidental appeal, code of civil procedure.

Recepción original: 04/06/2013

Aceptación original: 06/06/2013

Sommario: I. Le impugnazioni incidentali nel processo amministrativo italiano. II. Le modifiche strutturali e funzionali del processo prodotte dalla proposizione dell'impugnazione incidentale. III. Il principio di concentrazione delle impugnazioni. IV. Il campo di applicazione dell'onere di proporre l'impugnazione successiva alla prima in via incidentale. V. La proposizione delle impugnazioni successive alla prima deve avvenire con le forme previste per le impugnazioni incidentali a pena di decadenza. VI. L'impugnazione incidentale tardiva. VII. L'impugnazione incidentale tardiva innescata dall'integrazione del contraddittorio. VIII. Il rapporto di condizionalità tra l'impugnazione principale e l'impugnazione incidentale. IX. Il problema della proponibilità dell'impugnazione incidentale solo avverso lo stesso capo di sentenza oggetto dell'impugnazione principale oppure anche avverso capi autonomi. X. L'ordine di esame delle impugnazioni. XI. L'impugnazione incidentale proposta fuori termine non può digradare ad atto di intervento. XII. Gli effetti dell'onere di proporre l'impugnazione successiva alla prima in via incidentale sui termini per impugnare. XIII. Conclusioni.

I. Nel processo amministrativo italiano le impugnazioni incidentali sono state oggetto di una disciplina espressa solo a seguito dell'emanazione del codice del processo amministrativo. Prima dell'emanazione del codice le uniche disposizioni sulle impugnazioni incidentali nel processo amministrativo erano quelle previste dall'art. 22 t. u. 1058/1924 e dall'art. 37 t. u. 1054/1924 per il ricorso 
incidentale innanzi al Consiglio di Stato, quale giudice di primo e unico grado, che in virtù dell'art. 29 l.n. 1034/1971 disciplinavano l'appello incidentale. Pertanto, in un primo e non breve periodo, l'unica figura di impugnazione ammessa nel processo amministrativo era l'appello incidentale ${ }^{1}$ e il parametro di riferimento era costituito dal ricorso incidentale in primo grado ${ }^{2}$.

1 Sull'appello incidentale si veda Cons. Stato, Ad. plen., 22.12.1982 n. 21, con nota di F. LUBRANO, Osservazioni in tema di appello incidentale, in Dir. proc. amm., 1984, 237 ss.; Cons. Stato, VI, 25.02.1989 n. 173, con nota di D. ARDINI, Ancora un tentativo di unificazione delle opposte tesi in materia di appello incidentale, in Dir. proc. amm., 1989, 453 ss.

2 Sul ricorso incidentale nel processo amministrativo, si vedano L. RAGGI, Il ricorso incidentale nella nostra giustizia amministrativa, in Rass. Dir. pubbl., 1953, I, 235 ss.; E. CAPACCIOLI, In tema di ricorso incidentale nel giudizio amministrativo, in Giur. compl. Cass. civ., 1951, II, 1014 ss.; A. PIRAS, Interesse legittimo e giudizio amministrativo, Milano, 1962, I, 204 ss.; A. M. SANDULLI, Il giudizio davanti al Consiglio di Stato, Napoli, 1963, 361 ss.; F. LUBRANO, L'impugnazione incidentale nel giudizio amministrativo, in Rass. Dir. pubbl., 1964, 756 ss.; A. PAJNO, Appunti a proposito del ricorso incidentale condizionato nel processo amministrativo, in Giur. di merito, 1975, IV, 110 ss.; R. MARRAMA, Rinuncia all'impugnazione ed acquiescenza al provvedimento amministrativo, Padova, 1987, 58 ss.; V. CAIANIELLO, Manuale di diritto processuale amministrativo, cit., 648 ss.; V. CAIANIELLO, Lineamenti del processo amministrativo, Torino, 1979, 397 ss.; F. LA VALLE, L'impugnazione incidentale del provvedimento amministrativo, in Riv. dir. proc., 1968, 544 ss.; G. ROEHRSSEN, Ricorso giurisdizionale amministrativo, in Nss. dig. it., XV, 1968, 1016 ss.; W. CATALLOZZI, Note sulle impugnazioni incidentali nel processo dinanzi ai giudici amministrativi ordinari, in Studi per il centocinquantenario del Consiglio di Stato, III, Roma, 1981, 1759 ss.; F. BENVENUTI, Contraddittorio (diritto amministrativo), in Enc. dir., IX, Milano, 1961, 744; G. VACIRCA, Appunti per una nuova disciplina dei ricorsi incidentali nel processo amministrativo, in Dir. proc. amm., 1986, 57 ss.; S. SANTORO, Appunti sulle impugnazioni incidentali nel processo amministrativo, in Dir. proc. amm., 1986, 424; E. PICOZZA, Processo amministrativo (normativa), in Enc. dir., XXXVI, 1987, 485 ss.; S. BACCARINI, L'impugnazione incidentale del provvedimento amministrativo fra tradizione e innovazione, in Dir. proc. amm., 1991, 633 ss.; G. ACQUARONE, In tema di rapporto tra ricorso principale e ricorso incidentale, nota a C.G.A., 22 dicembre 1995, n. 388, in Dir. proc. amm., 1997, 555 ss.; W. CATALLOZZI, Ricorso incidentale (Giudizio amministrativo), in Enc. giur. Treccani, XXVII, Roma, 1991; C.E. GALLO, Ricorso incidentale. Commento all'art. 22 L. 6 dicembre 1971, n. 1034, in A. ROMANO (a cura di) Commentario breve alle leggi di giustizia amministrativa, Padova, 2001, 825 ss.; M.A. RUSSO, La nozione di incidentalità nel campo amministrativo: ricorso incidentale e appello incidentale, in Dir. e formazione, 2003, 1473 ss.; G. ABBAMONTE, R. LASCHENA, Giustizia amministrativa, in Trattato di diritto amministrativo, diretto da Santaniello, XX, Padova, 2001, 297 ss.; D. TRAINA, Lo svolgimento del processo, in Trattato di diritto amministrativo, a cura di S. CASSESE, Diritto amministrativo speciale, IV, Il processo amministrativo, Milano, 2000, 3320 ss.; P. TROIANO, Il ricorso incidentale, in G. MORBIDELLI (a cura di ) Codice della giustizia amministrativa, Milano, 2005, 619 ss.; R. GIOVAGNOLI, Il ricorso incidentale, in Trattato di giustizia amministrativa. Il processo amministrativo di primo grado, a cura di F. CARINGELLA-R. GAROFOLI, Milano, 2005, 705 ss.; G. FERRARA, Il ricorso incidentale nel processo amministrativo: principi consolidati e problematiche irrisolte, in Dir. proc. amm., 2007, 1058 ss.; R. GIOVAGNOLI, M. FRATINI, Il ricorso incidentale e i motivi aggiunti, Milano, 2008; R. GRECO, I profili processuali del ricorso 
Sennonché, l'innesto dell'appello incidentale sul tronco rappresentato dalla disciplina del ricorso incidentale provocava una serie di rilevanti conseguenze. Invero, l'art. 37, sesto comma, t. u. 1054/1924 stabiliva che «il ricorso incidentale non è efficace, se venga prodotto dopo che si sia rinunziato al ricorso principale, o se questo venga dichiarato inammissibile, per essere stato proposto fuori termine». Pertanto, l'unico modello di appello incidentale, analogamente a quanto avveniva per il ricorso incidentale in primo grado, era solo quello legato da un nesso di accessorietà e subordinazione all'atto introduttivo del processo, vale a dire all'appello principale 3 .

Ne deriva che l'unica figura di impugnazione incidentale conosciuta, o comunque la principale impugnazione nota, era quella denominata impugnazione incidentale propria, vale a dire quella fondata su una situazione di soccombenza formale.

La prevalenza dell'impugnazione incidentale propria, nel panorama complessivo del processo amministrativo, costituisce una conseguenza diretta della derivazione della sua disciplina da quella dettata per il ricorso incidentale. La predetta derivazione costituisce il peccato originale che connoterà l'intera storia dell'istituto, concentrando la riflessione su profili marginali.

Invero, il ricorso incidentale viene esperito dalla parte che versi in una situazione di momentanea insussistenza dell'interesse a ricorrere, destinata ad attualizzarsi solo a seguito della proposizione del ricorso principale. Inoltre, tale condizionamento dell'interesse a ricorrere dalla proposizione del ricorso principale lega il ricorso incidentale a quest'ultimo da un nesso di condizionalità dipendenza, codificato dall'art. 37, sesto comma, t. u. n. 1054/1924.

Siffatte caratteristiche sono state attribuite anche all'impugnazione incidentale, con conseguente rilevanza esclusiva della figura della impugnazione incidentale propria.

Sennonché, la presenza nel contenzioso giurisdizionale amministrativo di processi litisconsortili con pluralità di parti soccombenti pose il problema della sorte da riservare alle impugnazioni successive alla prima, proposte da soggetti che si trovavano in uno stato originario di soccombenza pratica o sostanziale ${ }^{4}$.

incidentale, 2009, 629 ss.; A. ROMANO TASSONE, Il ricorso incidentale e gli strumenti di difesa nel processo amministrativo, in Dir. proc. amm., 2009, 581 ss.

3 In tal senso S. PERONGINI, Le impugnazioni nel processo amministrativo, Milano, 2011, 79.

4 Sulla soccombenza sono state prospettate diverse interpretazioni. Sulla soccombenza come interesse a impugnare L. MORTARA, Commentario del codice e delle 
L'esigenza di assicurare in maniera più ampia possibile la concentrazione delle impugnazioni condusse dottrina e giurisprudenza, dopo non pochi tentennamenti, a ritenere applicabile anche al processo amministrativo le disposizioni dettate dal codice del processo civile per le impugnazioni incidentali ${ }^{5}$. Nel vigente sistema processuale civile, l'impugnazione proposta per prima assume la denominazione e la veste di impugnazione principale, mentre tutte le impugnazioni successive assumono quelle di impugnazione incidentale.

Si diffuse un orientamento che, recependo la nozione di incidentalità accolta dal codice di procedura civile del 1940, impose anche per il processo amministrativo, a ogni soggetto destinatario della notificazione di un appello, di proporre la propria impugnazione in via incidentale, qualunque fosse la sua posizione, vale a dire autonoma, adesiva o contrastante rispetto a quella fatta valere dall'appellante principale $^{6}$. Ne derivò l'esigenza di esperire le impugnazioni suc-

leggi di procedura civile, Milano, s.d. ma 1908, II, 594; G. CHIOVENDA, Istituzioni di diritto processuale civile, Napoli, I, 1935, 534 ss.; E. BETTI, Diritto processuale civile italiano, Roma, 1936, 638; E. REDENTI, Diritto processuale civile, Milano, 1957, n. 146; R. PROVINCIALI, Delle impugnazioni in generale, Napoli, 1962, 125 ss.; U. ROCCO, Trattato di diritto processuale civile, III, Torino, 1966, 293; E. GRASSO, Le impugnazioni incidentali, Milano, 1973, 85 ss.. Sulla soccombenza come legittimazione a impugnare: E. ALLORIO, L'ordinamento giuridico nel prisma dell'accertamento giudiziale, Milano, 1957, 249; S. SATTA, Commentario al codice di procedura civile, II, Milano, 1959, 2, 18; E. FAZZALLARI, Il giudizio civile di cassazione, Milano, 1960, 37, 43 ss.. Sulla soccombenza indifferentemente come l'interesse ad agire e come la legittimazione P. CALAMANDREI, FURNO, Cassazione civile, in Nss. dig. it., II, 1958, 1066, 1069; S. CHIARLONI, L'impugnazione incidentale nel processo civile, Milano, 1969, 62. Identifica la soccombenza con un presupposto processuale G. A. MICHELI, Corso di diritto processuale civile, Milano, 1959, I, 19 ss., II, 240. Sul problema della soccombenza si vedano F. LANCELLOTTI, La soccombenza requisito di legittimazione alle impugnazioni, Milano, 1996, 14; S. PERONGINI, Le impugnazioni, op. cit., 2011, $26 \mathrm{ss}$.

5 Sulle impugnazioni incidentali nel processo civile, S. CHIARLONI, L'impugnazione incidentale nel processo civile, Milano,1969; E. GRASSO, Le impugnazioni incidentali, Milano, 1973; A. ATTARDI, Limiti di applicazione del gravame incidentale tardivo, in Riv. dir. proc., 1965, 173; A. ATTARDI, Sulle impugnazioni incidentali condizionate, in Giur. it., 1991, IV, 289; S. RECCHIONI, Note in tema di impugnazioni incidentali tardive e termine di impugnazione, in Giur. it., 1991, I, 2, 445; E.T. LIEBMAN, Arbitrarie limitazioni all'impugnazione incidentale, in Riv. dir. proc., 1969, 572; A. CHIZZINI, Ancora sui limiti di applicazione dell'impugnazione incidentale tardiva: la decisione delle sezioni unite, in Giur. it.,1990, I, 1, 391; A. CHIZZINI, Revirement della giurisprudenza in tema di impugnazioni incidentali tardive, in Giur. it., 1989, I, 1, 1135; GAMBINERI, orientamenti e disorientamenti giurisprudenziali in tema di limitazioni soggettive alla impugnazione incidentale tardiva, in Foro it., 1996, I, 919.

6 In tal senso, Cons. Stato, V, 15.12.1978 n. 1614; Cons. Stato, V, 19.10.1979 n. 584; Cons. Stato, IV, 28.08.1981 n. 677; Cons. Stato, IV, 21.06 .1982 n. 398; Cons. 
cessive alla prima rispettando la forma e i termini di proposizione dell'impugnazione incidentale ${ }^{7}$, vale a dire, per l'appello, le forme previste dall'art. 343 c.p.c. e, per il ricorso per cassazione, quelle disciplinate dall'art. 371 c.p.c.

Il codice del processo amministrativo ha disciplinato le impugnazioni incidentali all'art. 96, con una norma che innova notevolmente la materia ${ }^{8}$.

La norma, innanzi tutto, risolve il problema dell'individuazione della fonte normativa ${ }^{9}$, che ha occupato a lungo dottrina ${ }^{10}$ e giurisprudenza. Inoltre, essa consente di parlare di impugnazione incidentale e non solo di appello incidentale, come era nel precedente ordinamento $^{11}$.

L'art. 96, secondo comma, c.p.a. prevede che «possono essere proposte impugnazioni incidentali, ai sensi degli articoli 333 e 334 del codice di procedura civile». A sua volta, l'art. 333 stabilisce che «le parti alle quali sono state fatte le notificazioni previste negli articoli precedenti debbono proporre, a pena di decadenza, le loro impugnazioni in via incidentale nello stesso processo». Inoltre, l'art. 334 c.p.c. prevede che «Le parti, contro le quali è stata proposta impugnazione e quelle chiamate a integrare il contraddittorio a nor-

Stato, IV, 25.03.1983 n. 165; Cons. Stato, VI, 13.05 .1985 n. 197; Cons. Stato, IV, 1.08.1985 n. 327; Cons. Stato, VI, 25.02.1989 n. 173; Cons. Stato, V, 30.09.1992 n. 904.

7 Un consistente orientamento sosteneva che l'appello successivo al primo, anche se proposto da una parte portatrice di un interesse autonomo a impugnare, dovesse assumere la forma dell'appello incidentale ed essere articolato nei relativi termini, rappresentati da trenta giorni, dopo la scadenza del termine per il deposito dell'appello principale, e da sessanta giorni dalla notifica della sentenza nell'impugnazione di un capo autonomo. In tal senso, Cons. Stato, VI, 22.01.2001 n. 366; Cons. Stato, VI, 25.07.2001 n. 4077; Cons. Stato, VI, 9.05.2002 n. 2537; Cons. Stato, VI, 9.07.2004 n. 5025; Cons. Stato, VI, 7.10.2004 n. 6501; Cons. Stato, VI, 31.01.2005 n. 217; Cons. Stato, VI, 3.02.2005 n. 281;

8 Sulle impugnazioni incidentali, secondo il codice del processo amministrativo, si vedano E. CASETTA, Manuale di diritto amministrativo, XII, Milano, 2010, 908 ss.; A. TRAVI, Lezioni di giustizia amministrativa, IX, Torino, 2010, 307 ss.; S. PERONGINI, Le impugnazioni nel processo amministrativo, Milano, 2011, 77 ss.; A. ZITO, Le impugnazioni, in F.G. SCOCA (a cura di), Giustizia amministrativa, Torino, 2013, 428 ss.

9 Secondo V. CAIANIELLO, Manuale, 2003, 920, la fonte normativa va individuata nell'art. 37 t.u. n. 1054/1924, in forza del rinvio operato dall'art. 29, primo comma, l.n. 1034/1071.

10 G. LEONE, Il sistema, 2006, 284, sostiene che la fonte dell'impugnazione incidentale vada individuata nell'art. 333 c.p.c. e nei principi desumibili dall'ordinamento processuale civile. In termini, si veda Cons. Stato, VI, 15.02.1989 n. 173.

11 In tal senso S. PERONGINI, Le impugnazioni nel processo amministrativo, Milano, 2011, 81. 
ma dell'art. 331, possono proporre impugnazione incidentale anche quando per esse è decorso il termine o hanno fatto acquiescenza alla sentenza. In tal caso, se l'impugnazione principale è dichiarata inammissibile, la impugnazione incidentale perde ogni efficacia».

Per effetto del richiamo effettuato dall'art. 96, secondo comma, c.p.a., anche gli artt. 333 e 334 c.p.c. trovano applicazione diretta nel processo amministrativo.

Tuttavia, il codice del processo amministrativo, come già messo in evidenza da una dottrina ${ }^{12}$, presenta significative novità rispetto alle disposizioni previgenti al codice e rilevanti differenze rispetto alle stesse disposizioni dettate dal codice di procedura civile.

Innanzi tutto, con il codice del processo amministrativo scompare la disciplina specifica prevista per il ricorso incidentale innanzi al Consiglio di Stato (alias appello incidentale) e viene sostituita da una disciplina generale, valida per tutte le impugnazioni incidentali ${ }^{13}$.

Inoltre, il codice del processo amministrativo determina un vero e proprio capovolgimento del rapporto tra impugnazione incidentale propria e impugnazione incidentale impropria ${ }^{14}$. Prima dell'emanazione del codice, l'art. 22 t. u. 1058/1924, l'art. 37 t. u. 1054/1924 e l'art. 29 l.n. 1034/1971 disciplinavano l'appello incidentale proprio. La giurisprudenza considerava del tutto eccezionale e residuale la figura dell'appello incidentale improprio. Solo negli anni 80 , la giurisprudenza ha cominciato a configurare l'appello incidentale improprio come ipotesi ordinaria.

Invece, l'art. 96 c.p.a. disciplina l'appello incidentale improprio e l'appello incidentale tardivo, omettendo ogni espresso riferimento all'appello incidentale proprio. In particolare l'art. 96, secondo comma, c.p.a. sancisce che "possono essere proposte impugnazioni incidentali, ai sensi degli artt. 333 e 334 del codice di procedura civile».

In virtù della succitata disposizione, tutte le parti destinatarie della notifica dell'impugnazione, devono proporre, a pena di decadenza, le loro impugnazioni in via incidentale ${ }^{15}$.

La disciplina introdotta dal codice del processo amministrativo pone i seguenti problemi: a) individuare il campo di riferimento della disciplina delle impugnazioni incidentali nel processo amministrativo e, in particolare, verificare per quali mezzi di impugnazione si

12 Cfr., S. PERONGINI, Le impugnazioni, 2011, 82.

13 Cfr., S. PERONGINI, Le impugnazioni, 2011, 82.

14 Cfr., S. PERONGINI, Le impugnazioni, 2011, 82 ss.

15 Cfr., S. PERONGINI, Le impugnazioni, 2011, 82. 
applichino le norme dettate dal codice del processo amministrativo per le impugnazioni incidentali; b) precisare non quali mezzi di impugnazione il soccombente "possa" proporre in forma incidentale, bensì quali mezzi «debba» proporre in forma incidentale, secondo quanto disposto dagli artt. 96 c.p.a. e 333, 334 c.p.c., specificando se la proposizione delle impugnazioni successive alla prima debba avvenire con le forme previste per le impugnazioni incidentali a pena di decadenza; c) verificare quali connotazioni possegga l'impugnazione incidentale tardiva, in che termini sia proponibile e se il riferimento, contenuto nel codice del processo amministrativo, alla categoria delle impugnazioni incidentali tempestive e a quelle tardive abbia condotto all'elisione delle impugnazioni incidentali così dette proprie; d) verificare se intercorra un rapporto di condizionalità tra l'impugnazione principale e l'impugnazione incidentale e quali connotazioni detto rapporto possegga; e) precisare se l'impugnazione incidentale debba essere rivolta verso lo stesso capo di sentenza oggetto dell'impugnazione principale oppure anche avverso capi autonomi; f) precisare l'ordine di esame delle impugnazioni; g) ricostruire gli effetti dell'onere di proporre impugnazione successiva alla prima in via incidentale sui termini per impugnare; h) infine, accertare se l'impugnazione incidentale fuori termine possa digradare ad intervento.

I predetti problemi dovranno essere affrontati nei paragrafi che seguono.

II. Innanzi tutto, si deve evidenziare che l'impugnazione incidentale modifica la struttura di base del processo amministrativo classico di impugnazione, in maniera analoga all'alterazione della struttura classica del processo di impugnazione prodotta dalla proposizione del ricorso incidentale in primo grado. La modifica strutturale incide anche sui profili funzionali del processo, connotandoli in maniera originale.

Invero, la proposizione del ricorso incidentale o della impugnazione incidentale sottrae, rispettivamente, al ricorrente o al soccombente che ha proposto il ricorso o l'impugnazione principale, l'esclusiva del potere di impulso processuale.

Il ricorrente e l'impugnante principale, normalmente domini dell'andamento processuale, titolari dell'iniziativa processuale e dei correlativi poteri di impulso, si vedono sottratta l'esclusiva o, comunque, la primazia del potere di impulso processuale, il quale viene distribuito diversamente da quanto avvenga nel processo che vede contrapposto privato e pubblica amministrazione. Inoltre, essi 
subiscono l'iniziativa processuale del controinteressato con il ricorso incidentale e, per quanto ci riguarda, del soccombente con l'impugnazione incidentale.

Con la proposizione del ricorso incidentale o della impugnazione incidentale, l'oggetto del giudizio si amplia. Accanto alle questioni sollevate con il ricorso principale in primo grado o con l'impugnazione principale, il giudice deve esaminare e decidere anche le questioni di fatto o di diritto proposte con il ricorso incidentale o con la impugnazione incidentale.

Nel processo arricchito dalla proposizione del ricorso incidentale in primo grado o della impugnazione incidentale, il rapporto processuale si implementa. Esso non risulta costituito solo dalla relazione che lega ricorrente e amministrazione, secondo un orientamento, e giudice, ricorrente e amministrazione, secondo un altro, ma si arricchisce con l'instaurazione di tutta una fitta rete di relazioni processuali intercorrenti fra le diverse parti intervenute nel processo a seguito della proposizione del ricorso incidentale in primo grado o della impugnazione incidentale.

Anche l'istruttoria subisce gli effetti derivanti dalla presenza di più parti nel processo. Il materiale istruttorio si arricchisce con l'apporto derivante dal contributo dei nuovi protagonisti della vicenda processuale. L'apporto istruttorio di alcuno giova o nuoce anche agli altri; il principio dell'onere della prova, per la presenza di più parti, si articola diversamente da quanto avviene nel processo con due soli soggetti; il principio di non contestazione impone alle parti adempimenti più complessi e provoca effetti di accertamento della prova più articolati.

La proposizione del ricorso incidentale da parte del controinteressato altera la distribuzione dei poteri processuali fra giudice e parti e, in particolare, fra giudice, ricorrente e pubblica amministrazione. Non vi sono posizioni di esclusiva nella titolarità dei poteri processuali, ma questi vengono ripartiti fra tutti i soggetti presenti nel processo. La pubblica amministrazione non subisce soltanto un attacco frontale, ma è costretta a difendersi anche dalle incursioni che la colpiscono nelle retrovie. Il giudice si trova di fronte tre contendenti le cui posizioni, quasi sempre, non coincidono e che sono fra di loro in aperto contrasto.

I poteri del giudice vengono esercitati nei confronti di una pluralità di parti e vanno contemperati con tutte le esigenze processuali 
derivanti dalla contemporanea presenza di più protagonisti della vicenda processuale.

La proposizione del ricorso incidentale in primo grado o della impugnazione incidentale determina l'applicazione di norme e principi processuali che incidono sulla statica e sulla dinamica del processo. Si pensi, a tal proposito, all'applicazione del principio di concentrazione delle impugnazioni e dei correlati poteri che, dagli istituti che del suddetto principio costituiscono applicazione, derivano ai soggetti e alle parti del processo. Su questi profili bisognerà ritornare più oltre.

L'ordine di esame e di definizione delle questioni subisce una profonda e radicale trasformazione rispetto all'ordine che il giudice è tenuto a seguire, sulla scorta di principi logici e di principi giuridici, nel diverso caso in cui il processo si svolga solo con la presenza del ricorrente e dell'amministrazione.

Anche la funzione del processo subisce un'evoluzione, sottile, impalpabile, quanto si voglia, ma pur sempre tale da consentire di rimarcare le differenze.

L'alterazione del modello tipico del processo di impugnazione indotta dal ricorso incidentale e dall'impugnazione incidentale è stata segnalata da L. Mortara, in un suo scritto del 1904.

Tuttavia, l'indicazione non ha avuto la dovuta attenzione che meritava le venisse dedicata. In questa sede si tenterà di offrire qualche elemento di riflessione. A dir il vero, l'esame dei profili problematici delle impugnazioni incidentali, succintamente passati in rassegna nel precedente paragrafo, costituisce l'occasione per avviare la riflessione sulle modifiche strutturali e funzionali del processo prodotte dalla proposizione dell'impugnazione incidentale, naturalmente nei limiti naturali di un saggio dedicato ad altri aspetti dell'istituto in esame.

III. Prima di affrontare i problemi sopra prospettati appare opportuno formulare qualche considerazione di ordine generale.

In primo luogo, si deve precisare che l'istituto delle impugnazioni incidentali assicura la concentrazione delle impugnazioni in un unico processo, la loro trattazione unitaria e la loro decisione con un'unica sentenza.

Il principio di concentrazione dei giudizi di impugnazione trova applicazione da tempo anche nel processo amministrativo. Infatti, l'art. 52 r.d. 642/1907 dettava una disciplina particolare per la riu- 
nione $^{16}$ e la giurisprudenza, seppur fra alterne posizioni, sosteneva l'applicabilità anche al processo amministrativo dei principi dettati in materia dal codice del processo civile.

Oggi la materia è oggetto di una disciplina specifica.

Il principio di concentrazione dei giudizi di impugnazione trova applicazione in quattro istituti giuridici, due in via preventiva e due in via successiva ${ }^{17}$. Con l'espressione «applicazione in via preventiva del principio» si fa riferimento agli effetti che il principio esplica su istituti che concernono la fase prodromica del processo. Invece, con la locuzione «applicazione in via successiva del principio» si fa riferimento agli effetti prodotti su istituti relativi al processo già instaurato.

Il principio di concentrazione dei giudizi di impugnazione, in via preventiva ${ }^{18}$, vive nell'art. 95, primo comma, c.p.a., il quale sancisce che l'impugnazione va notificata, in "causa inscindibile o in cause tra loro dipendenti», a tutte le parti in causa e, «negli altri casi», a tutte quelle che hanno interesse a contraddire.

Come è noto, la prima formulazione dell'art. 95, primo comma, distingueva fra "cause inscindibili» e «altri casi». A tal proposito, una dottrina ha avuto modo di precisare che la locuzione «cause inscindibili» è anomala perché il termine «inscindibilità» indica che la materia del contendere è così intimamente fusa, che non è possibile operare una separazione ${ }^{19}$. Inoltre, la predetta locuzione fa riferimento all'area delle fattispecie comprese nel novero della nozione di "causa inscindibile» e non è idonea a indicare altre fattispecie, coincidendo integralmente con la prima ${ }^{20}$. In altri termini, la sostituzione della locuzione "causa inscindibile», utilizzata nell'art. 331 c.p.c., con quella di «cause inscindibili», presente nella versione originaria dell'art. 95 c.p.a. rappresentava una contraddizione in termini, che

16 L'art. 52 r.d. 642/1907, abrogato dall'art. 4, primo comma, numero 2, dell'allegato 3 al c.p.a., prevedeva che «se alcuna delle parti o la pubblica amministrazione chieda che per ragioni di connessione due ricorsi siano uniti e venga provveduto su di essi con una sola decisione, la sezione, udite le parti interessate, può ordinarne l'unione. Il Presidente può, anche quando non sia stata chiesta l'unione, ordinare di ufficio che i due ricorsi siano chiamati di ufficio alla stessa udienza, affinché la sezione possa giudicare della loro connessione e, ove si faccia luogo alla riunione, pronunciare sui due ricorsi con una sola decisione».

17 Sul tema, S. PERONGINI, Le impugnazioni nel processo amministrativo, Milano, 2011, 46 ss.

18 Sul tema, S. PERONGINI, Le impugnazioni nel processo amministrativo, Milano, 2011, 49.

19 Cfr., S. PERONGINI, Le impugnazioni nel processo amministrativo, Milano, 2011, 62.

20 Cfr., S. PERONGINI, Le impugnazioni, 2011, 63. 
non implicava una diversa efficacia normativa, ma costituiva l'evidente frutto di una disattenzione del legislatore.

L'orientamento in esame ha evidenziato che le «cause dipendenti» non sono «cause inscindibili» e non trovano idonea collocazione nella predetta categoria ${ }^{21}$ e non rientrano neppure nell'ambito della categoria delimitata dalla locuzione «altri casi». Negli «altri casi» possono rientrare le "cause scindibili», ma non le "cause dipendenti», con la conseguenza che le fattispecie configurate dalla nozione di «cause dipendenti» non trovano collocazione né nella prima, né nella seconda categoria elaborata dal codice del processo amministrativo ${ }^{22}$.

Pertanto, la tripartizione di cause, elaborata dalla dottrina processualicivilistica e recepita dal codice di procedura civile, non riusciva a rinvenire nel codice del processo amministrativo idonea collocazione, con la conseguenza che alcune tipologie di cause (segnatamente le cause dipendenti) non trovavano alcuna disciplina.

Il legislatore, in sede di primo correttivo al codice del processo amministrativo, ha eliminato l'errore in cui era incorso riproducendo la formulazione adottata dagli artt. 331 e 332 c.p.c. e distinguendo, pertanto, da un lato, la «causa inscindibile» e le «cause tra loro dipendenti»e, dall'altro, le «cause scindibili» ${ }^{23}$.

Il principio di concentrazione delle impugnazioni si estrinseca in via preventiva anche nell'onere di proporre le impugnazioni, successive alla prima, in via incidentale. L'applicazione di detto principio in via preventiva comporta che le impugnazioni successive alla prima debbano essere formulate in via incidentale ${ }^{24}$.

Invece, il potere conferito al giudice di procedere alla riunione dei ricorsi è manifestazione successiva del principio di concentrazione in esame ${ }^{25}$.

Infatti, l'art. 96, primo comma, c.p.a. rubricato «impugnazioni avverso la medesima sentenza», stabilisce che "tutte le impugnazioni proposte separatamente contro la stessa sentenza devono essere riunite in un solo processo».

L'art. 96, primo comma, riproduce l'art. 335 c.p.c., a esclusione dell'espressione "anche di ufficio». Si è sostenuto che la predetta omissione non debba essere interpretata come una consapevole

21 Cfr., S. PERONGINI, Le impugnazioni, 2011, 63 ss.

22 Cfr., S. PERONGINI, Le impugnazioni, 2011, 63 ss.

23 Sul tema, S. PERONGINI, Le impugnazioni, 2011, 60 ss.

24 Sul tema, S. PERONGINI, Le impugnazioni, 2011, 57 ss., 77 ss.

25 Sul tema, S. PERONGINI, Le impugnazioni, 2011, 89. 
scelta volta a evitare che la riunione delle impugnazioni venga disposta anche di ufficio e volta ad assoggettare la materia al principio dispositivo, devolvendo solo alle parti il potere di decidere se riunire o meno le impugnazioni ${ }^{26}$.

Secondo la tesi in esame, la mancanza nell'art. 96, primo comma, c.p.a. dell'espressione «anche di ufficio», presente, invece, nell'art. 335 c.p.c., non incide sui poteri del giudice di disporre la riunione delle impugnazioni, che possono essere esercitati «anche di ufficio» ${ }^{27}$.

Secondo l'orientamento in esame, la riunione dei processi è sottratta alla disponibilità delle parti e rientra nella tecnica del pro$\operatorname{cesso}^{28}$. La riunione, infatti, è finalizzata ad assicurare la più breve durata del processo e soddisfa esigenze di economia processuale. La definizione congiunta dei processi riduce il tempo di definizione delle controversie, in ossequio al principio costituzionale del giusto processo $^{29}$. A tal proposito, si consideri che il codice del processo amministrativo sancisce il principio del giusto processo (art. 2, primo comma, c.p.a.) e impone al giudice e alle parti di cooperare per assicurare una durata ragionevole al processo (art. 2, secondo comma, c.p.a.). Soprattutto quest'ultima norma si traduce nel conferimento al giudice di tutti i poteri necessari per ridurre i tempi di svolgimento del processo. Fra detti poteri rientra a pieno titolo anche quello di disporre la riunione di più impugnazioni proposte avverso la medesima sentenza. Inoltre, la riunione dei ricorsi riduce il rischio di giudicati contrastanti ${ }^{30}$.

Questo spiega perché la mancanza nell'art. 96, primo comma, c.p.a. dell'espressione "anche di ufficio», presente, invece, nell'art. 335 c.p.c., non muta la sostanza delle cose, trattandosi sempre di esercizio del potere ordinatorio del giudice, preposto alla direzione del processo $^{31}$.

26 Sul tema, S. PERONGINI, Le impugnazioni, 2011, 47.

27 In tal senso, M. LIPARI, Impugnazione in generale, in A. QUARANTA, V. LOPILATO (a cura di), Il processo amministrativo, Milano, 2011, 723; S. PERONGINI, Le impugnazioni, 2011, 47.

28 S. PERONGINI, Le impugnazioni, 2011, 48.

29 Sul tema, G. CARLOTTI, La riunione dei ricorsi e l'integrazione del contraddittorio, in G. CARLOTTI, M. FRATINI, L'appello al Consiglio di Stato, Milano, 2008, 612; S. PERONGINI, Le impugnazioni, 2011, 48.

30 G. CARLOTTI, La riunione dei ricorsi e l'integrazione del contraddittorio, in G. CARLOTTI, M. FRATINI, L'appello, 2008, 612, 615; S. PERONGINI, Le impugnazioni, 2011, 48.

31 In tal senso, S. CASSARINO, Manuale di diritto processuale amministrativo, Milano, 1990, 491; G. CARLOTTI, La riunione dei ricorsi, 2008, 615; S. PERONGINI, Le impugnazioni, 2011, 48. 
Infine, il principio di concentrazione dei giudizi di impugnazione trova applicazione, in via successiva, anche nell'art. 95, terzo comma, c.p.a. il quale conferisce al giudice il potere di integrare il contraddittorio quando la sentenza non sia stata impugnata nei confronti di tutte le parti ${ }^{32}$.

La concentrazione delle impugnazioni in via preventiva è assicurata dalla previsione di specifici oneri in capo alle parti; mentre, in via successiva, è garantita dal conferimento al giudice di una serie di poteri ${ }^{33}$.

In secondo luogo, appare opportuno premettere che l'indagine consentirà di verificare se e in che misura la soluzione ai numerosi problemi sopra prospettati dipende dal grado di intensità con il quale si intende attuare il principio di concentrazione delle impugnazioni. La soluzione offerta, anche in passato, dalla giurisprudenza e dalla dottrina ai problemi sopra prospettati rispecchia il grado di intensità in cui si è inteso applicare il principio di concentrazione delle impugnazioni e, in particolare, la decisione di attuarlo in maniera rigida $\mathrm{o}$ in maniera blanda.

IV. Uno dei primi problemi da affrontare è quello di individuare il campo di applicazione delle impugnazioni e, in particolare, i mezzi di impugnazione per i quali sia proponibile anche l'impugnazione in via incidentale. Invero, il tema è stato oggetto di approfondite indagini nel processo civile ${ }^{34}$, sollecitate dal fatto che nel relativo codice, pur essendo presente una disciplina generale per le impugnazioni incidentali (artt. 333, 334 c.p.c.), astrattamente efficace per ogni mezzo di impugnazione, sono disciplinati in maniera specifica solo l'appello incidentale (art. 343 c.p.c.) e il ricorso incidentale per cassazione (art. 371 c.p.c.).

La dottrina processualcivilista sostiene che per ognuno dei quattro mezzi di impugnazione può proporsi l'impugnazione incidentale ${ }^{35}$.

Il problema succitato va posto anche per il processo amministrativo. L'analisi, delle disposizioni dedicate dal codice del processo amministrativo all'istituto in esame, consente di individuare una

\footnotetext{
32 S. PERONGINI, Le impugnazioni, 2011, 90 ss.

33 S. PERONGINI, Le impugnazioni, 2011, 48.

34 Sul tema S. CHIARLONI, L'impugnazione incidentale nel processo civile, Milano, 1969, 4 ss.

35 Sostengono la proponibilità dell'impugnazione incidentale per tutti i mezzi di impugnazione N. GIUDICEANDREA, Le impugnazioni civili, Milano, 1952, 235; G.A. MICHELI, Corso di diritto processuale civile, Milano, 1960, 253; M. VELLANI, Appunti sulla impugnazione incidentale tardiva, in Riv. trim. dir. proc. civ., 1951, 991; M.T. ZANZUCCHI, Diritto processuale civile, II, Milano, 19962, 199.
} 
serie di ragioni che inducono a ritenere ammissibile l'impugnazione incidentale in presenza di ogni mezzo di impugnazione.

La possibilità di proporre l'impugnazione incidentale in presenza di ogni mezzo di impugnazione si desume dalla collocazione della norma che contempla le impugnazione incidentali (art. 96 c.p.a.) nel libro terzo, titolo primo, dedicato alle impugnazioni in generale. Trattandosi di una disciplina generale, tutti i mezzi di impugnazione possono essere proposti anche in via incidentale.

In tal senso, depone anche l'art. 96 c.p.a., in cui si parla sempre di impugnazioni al plurale e, soprattutto, si pone una disciplina che non contiene alcuna indicazione volta a precludere l'impugnazione incidentale per qualche mezzo particolare di impugnazione.

Infine, la disciplina specifica dettata dal codice del processo amministrativo per l'appello, per la revocazione, per l'opposizione di terzo e per il ricorso amministrativo non contiene alcuna espressa preclusione.

Tuttavia, la predetta prima impressione è destinata a scomparire in presenza di una più attenta esegesi.

Nessun dubbio che nell'appello possano essere proposte impugnazioni incidentali.

Invece, a una siffatta conclusione non può pervenirsi in ogni ipotesi in cui venga proposto ricorso per cassazione.

Il rinvio operato dall'art. 39 c.p.a. alle disposizioni del codice di procedura civile rende applicabile la disciplina contemplata per il ricorso incidentale in Cassazione dall'art. 371 c.p.c. anche al processo amministrativo. Tuttavia, occorre tener presente che nel processo amministrativo il ricorso per cassazione avverso le sentenze del Consiglio di Stato è limitato alle sole questioni di giurisdizione, ai sensi dell'art. 111, ultimo comma, della Costituzione e dell'art. 110 c.p.a.

In caso di pluralità di soccombenti, dopo la proposizione del ricorso da parte di uno di loro, gli altri non solo potranno proporre ricorso incidentale, ma dovranno procedere in tale senso per le ragioni che saranno successivamente svolte.

Questo vale per il ricorso incidentale in cassazione c.d. improprio, cioè quello proposto dai litisconsorti soccombenti sostanziali, ma può valere per il ricorso c.d. proprio, vale a dire quello proposto dal soccombente formale. Invero, la limitazione della proponibilità deriva dal fatto che il ricorso per cassazione avverso le sentenze del Consiglio di Stato è limitato alle sole questioni di giurisdizione. 
Sulla sola questione di giurisdizione non è possibile configurare una soccombenza formale. Sulla questione di giurisdizione sono configurabili solo due posizioni: quella di chi afferma la giurisdizione del giudice amministrativo, vittorioso sul punto attesa la sentenza emanata, e quella di chi la nega, sul punto soccombente. Quest'ultimo proporrà ricorso per cassazione, impugnando la sentenza emanata dal Consiglio di Stato. La parte vittoriosa non avrà alcuna possibilità di proporre impugnazione incidentale c.d. propria, non avendo alcuna valida argomentazione per intervenire.

Pertanto, il ricorso incidentale per cassazione sarà proponibile solo in presenza di un processo con pluralità di parti soccombenti in punto di giurisdizione. Invece, qualora il processo veda due sole parti contrapposte sulla questione della giurisdizione, non sarà configurabile alcun ricorso incidentale per cassazione, non essendo prospettabile, sulla stessa questione, una duplice soccombenza.

Anche per l'opposizione di terzo deve ammettersi la possibilità che altri terzi, diversi dall'opponente, possano articolare la loro opposizione in forma incidentale inserendosi nel processo innescato dall'opposizione principale.

Analoga conclusione deve raggiungersi anche per la revocazione.

Sulla scorta di quanto precede si può affermare che l'impugnazione incidentale può essere proposta in caso di appello, di opposizione di terzo e di revocazione. Invece, nel ricorso per cassazione la questione è più complessa, perché le sentenze del giudice amministrativo, come già detto, possono essere impugnate in cassazione solo per le questioni di giurisdizione. Pertanto, l'impugnazione incidentale può essere proposta solo dai litisconsorti soccombenti sostanziali o materiali, non negli altri casi. Non è configurabile sulla questione di giurisdizione contemporaneamente una soccombenza sostanziale e una soccombenza formale, se non nel caso eccezionale in cui il giudice amministrativo abbia declinato la propria giurisdizione, sostenendo nel contempo che la questione non appartenga ad alcun giudice.

Tuttavia, la possibilità di proporre le impugnazioni successive alla prima in forma incidentale in tutti i mezzi di impugnazione non risolve il problema sopra prospettato. Invero, non si tratta di stabilire se in tutti i mezzi di gravame le impugnazioni successive alla prima "possano" proporsi in forma incidentale, bensì se «debbano" proporsi in tal modo. Il predetto problema non può trovare soluzione in questa fase della ricerca, bensì solo successivamente dopo aver messo a fuoco ulteriori caratteristiche dell'istituto in esame. 
V. Uno dei primi problemi da affrontare è quello volto a individuare le conseguenze che derivano dall'omessa proposizione in via incidentale delle impugnazioni successive alla prima. La questione riveste particolare importanza, anche perché la sua soluzione offre elementi per definire altri profili problematici dell'istituto in esame.

Invero, il problema sopra prospettato si pone perché l'art. 96, secondo comma, c.p.a. sancisce che "possono essere proposte impugnazioni incidentali, ai sensi degli artt. 333 e 334 del codice di procedura civile». Inoltre, il riferimento all'art. 333 c.p.c. viene effettuato anche dall'art. 96, terzo comma, c.p.a.. L'art. 333 stabilisce che «le parti alle quali sono state fatte le notificazioni previste negli articoli precedenti debbono proporre, a pena di decadenza, le loro impugnazioni in via incidentale nello stesso processo».

Il richiamo all'art. 333 c.p.c. pone il problema di stabilire se l'onere di proposizione dell'impugnazione in via incidentale sia previsto a pena di decadenza ${ }^{36}$. In altri termini, in virtù delle succitate disposizioni, occorre verificare se tutte le parti destinatarie della notifica dell'impugnazione principale devono proporre le loro impugnazioni in via incidentale.

Prima dell'emanazione del codice, la giurisprudenza sosteneva che il destinatario della notificazione dovesse proporre impugnazione incidentale a pena di decadenza. Tuttavia, secondo il succitato orientamento giurisprudenziale, poiché la sanzione della decadenza era finalizzata ad assicurare la concentrazione delle impugnazioni, quando per altra via si conseguiva lo stesso risultato, veniva automaticamente meno la necessità di dichiarare la decadenza. In particolare, secondo l'orientamento giurisprudenziale in esame, quando il giudice ordinava la riunione delle diverse impugnazioni, veniva meno la necessità di dichiarare la decadenza ${ }^{37}$. Invero, con la riunione delle diverse impugnazioni si conseguiva il fine di evitare una proliferazione dei processi autonomi, si assicuravano le esigenze di economia processuale e si scongiurava il pericolo di giudicati contrastanti. In altri termini, si conseguivano tutti e gli stessi effetti collegati alla proposizione dell'impugnazione in via incidentale.

36 Sul tema, S. PERONGINI, Le impugnazioni nel processo amministrativo, Milano, 2011, 85 ss.

37 In tal senso Cons. di Stato, V, 22.06.1996 n. 790; Cons. di Stato, V, 15.03.1993 n. 357; Cons. di Stato, V, 31.07.1998 n. 1146; Cons. di Stato, V, 3.02.2000 n. 661; Cons. di Stato, V, 22.04.1996 n. 474; in dottrina si veda V. CAIANIELLO, Diritto processuale amministrativo, Torino, 2003, 925. 
Dopo l'emanazione del codice del processo amministrativo, un orientamento ha sostenuto che la proposizione dell'impugnazione successiva alla prima in via incidentale non sia prescritta a pena di decadenza ${ }^{38}$.

A tal proposito, si è sostenuto ${ }^{39}$ - richiamando evidentemente l'orientamento giurisprudenziale maturato prima dell'emanazione del codice del processo amministrativo - che la riunione dei giudizi, disposta dal giudice amministrativo, precluda la declaratoria di decadenza. La preclusione deriverebbe dal fatto che la decadenza sia stata prevista al fine di assicurare la concentrazione delle impugnazioni, che con la riunione delle impugnazioni sarebbe altrimenti conseguita. Il raggiungimento della predetta finalità rappresenterebbe la causa preclusiva della comminatoria di decadenza.

Tuttavia, una siffatta impostazione sembra costituire un elegante espediente per aggirare il problema. Invero, seguendo la prospettazione tradizionale, il problema della sussistenza di un effetto decadenziale rimane irrisolto in tutti quei casi in cui il giudice non dispone la riunione dei processi. In ogni caso, rimane da dimostrare in che modo la riunione (che avviene a valle) possa incidere sulla decadenza (che avviene a monte), vale a dire che si verifica nel momento in cui il destinatario della notifica dell'impugnazione principale non propone la sua impugnazione in via incidentale.

Un orientamento ha sostenuto che il codice del processo amministrativo non ha voluto comminare la decadenza perché non la ha contempla espressamente. Detta tesi si basa sul rilievo che quando il codice ha voluto richiamare un tratto della disciplina delle impugnazioni nel processo civile, lo ha fatto espressamente, per esempio, menzionando la possibilità di impugnare anche altri capi della sentenza. Dall'impiego di una siffatta tecnica normativa nell'art. 96 c.p.a. si è dedotto che l'omesso riferimento alla sanzione della deca-

38 A. TRAVI, Lezioni di giustizia amministrativa, IX, 2010, 309, sostiene che il codice del processo amministrativo, pur richiamando espressamente l'art. 333 c.p.c., non sembra comminare alcuna decadenza, se una parte abbia proposto la propria impugnazione in via autonoma, anziché in via incidentale. In termini identici, A. TRAVI, Lezioni di giustizia amministrativa, X, 2012, 312. S. OGGIANU, Impugnazio$n i$, in E. PICOZZA (a cura di), Codice del processo amministrativo, Torino, 2010, 162, sembra sostenere che la violazione del principio, che impone la proposizione delle impugnazioni successive alla prima in via incidentale, non sia assistito da alcuna sanzione.

39 R. CHIEPPA, Il codice del processo amministrativo, Milano, 2010, 435. 
denza costituisca la necessaria conseguenza della decisione di escludere l'applicazione della sanzione della decadenza ${ }^{40}$.

Tuttavia, una siffatta tesi non sembra convincente.

Infatti, secondo un orientamento, il riferimento dell'art. 96, secondo e terzo comma, c.p.a. alla proponibilità di impugnazioni incidentali ai sensi dell'art. 333 c.p.c. implica l'operatività a tutto campo della predetta norma e, quindi, l'applicazione della sanzione della decadenza a tutte quelle impugnazioni, proposte dai destinatari della notifica dell'impugnazione in via principale, che siano state formulate in via autonoma e non nella prescritta forma incidentale ${ }^{41}$.

Invero, il tema merita un approfondimento non solo per la sua ricaduta pratica, ma anche per le sue implicazioni teoriche.

Al fine di delimitare i termini della questione appare opportuno precisare che il problema della decadenza non si pone per coloro che abbiano proposto l'impugnazione in via autonoma, dopo la proposizione di altra che non sia stata loro notificata. Invero, solo la notifica dell'impugnazione principale genera l'onere di proporre le successive impugnazioni in via incidentale.

Non ha alcun rilievo la circostanza che il litisconsorte abbia avuto altrimenti conoscenza dell'impugnazione principale, perché senza la notifica l'onere in questione non sorge. Non è possibile alcuna indagine, più o meno complessa, sugli stati psicologici del soccombente che propone l'impugnazione successivamente ad altra.

Invero, la norma che commina la decadenza è eccezionale rispetto a quella generale di favore per la proposizione delle azioni in primo e secondo grado. Le norme eccezionali non sono suscettibili di interpretazione analogica e, pertanto, non è possibile ricollegare la sanzione della decadenza a un presupposto di fatto diverso da quello espressamente indicato dal legislatore. Per queste ragioni solo la notifica dell'impugnazione principale genera l'onere di proporre le impugnazioni successive alla prima in via incidentale.

La tesi, secondo la quale l'omessa proposizione della impugnazione successiva alla prima in forma incidentale determina la decaden-

40 Rilievi critici a detto orientamento in S. PERONGINI, L'impugnazione, 2011, 85 ss.

41 M.A. MERCATI, Commento all'art. 96 c.p.a., in R. GAROFOLI, G. FERRARI (a cura di), Codice del processo amministrativo, Roma, 2010, 1378; S. PERONGINI, Le impugnazioni nel processo amministrativo, Milano, 2011, 85 ss.; M. LIPARI, Impugnazioni in generale, in A. QUARANTA, V. LOPILATO (a cura di), Il processo amministrativo, Milano, 2011, 723, 725. 
za della relativa impugnazione, trova un decisivo supporto nel fatto che l'art. 96, secondo comma, c.p.a. stabilisce che possono essere proposte impugnazioni incidentali ai sensi dell'art. 333 c.p.c.. Il riferimento all'art. 333 c.p.c. implica l'applicazione integrale della norma relativa, anche in quella parte in cui essa commina la decadenza.

Nessuna rilevanza possiede l'incipit dell'art. 96, secondo comma, c.p.a., secondo il quale «possono essere proposte impugnazioni incidentali». L'uso del verbo "potere» in luogo di quello "dovere» deriva solo dal fatto che il codice del processo amministrativo ha inteso fare riferimento alla astratta possibilità che un soggetto proponga l'impugnazione incidentale e non vale a conferirgli una facoltà di scelta fra la proposizione di una impugnazione autonoma, idonea a incardinare un autonomo processo, e la proposizione di una impugnazione incidentale. In altri termini, la formulazione presente nella norma succitata attribuisce una facoltà di scelta fra il proporre e il non proporre impugnazione e non una facoltà di scelta fra il proporla in via autonoma in forma incidentale e il proporla in un separato processo.

Infine, in tal senso milita una decisiva ragione sistematica, rappresentata dal fatto che il codice del processo in tema di impugnazioni delinea un sistema chiuso, ispirato integralmente al principio di concentrazione delle impugnazioni. Su detto aspetto si è già richiamata l'attenzione in precedenza.

Infatti, in precedenza si è richiamata una dottrina secondo la quale il principio di concentrazione dei giudizi di impugnazione trova applicazione in quattro istituti giuridici, due in via preventiva e due in via successiva ${ }^{42}$.

Il principio di concentrazione dei giudizi di impugnazione, in via preventiva, trova espressione nell'art. 95, primo comma, c.p.a., il quale sancisce che l'impugnazione va notificata, nelle "causa inscindibile o in cause tra loro dipendenti», a tutte le parti in causa e, «negli altri casi», a tutte quelle che hanno interesse a contraddire. Secondo la tesi in esame, sempre in via preventiva, il principio in parola si estrinseca nell'onere di proporre le impugnazioni, successive alla prima, in via incidentale ${ }^{43}$.

Invece, in via successiva, il principio di concentrazione delle impugnazioni trova espressione nel potere conferito al giudice di procedere alla riunione dei ricorsi di «tutte le impugnazioni proposte 46 ss.

S. PERONGINI, Le impugnazioni nel processo amministrativo, Milano, 2011,

43 S. PERONGINI, Le impugnazioni nel processo amministrativo, Milano, 2011, 77 ss. 
separatamente contro la stessa sentenza», nonché nel potere di integrare il contraddittorio quando la sentenza non sia stata impugnata nei confronti di tutte le parti ${ }^{44}$.

In definitiva, la concentrazione delle impugnazioni in via preventiva è assicurata dalla previsione di specifici oneri in capo alle parti; mentre, in via successiva, è garantita dal conferimento al giudice di una serie di poteri.

In tal modo il legislatore ha delineato un sistema chiuso, completo, vale a dire un sistema in cui il principio di concentrazione delle impugnazioni trova sempre completa, integrale e rigorosa applicazione.

Se in detto quadro normativo, la proposizione delle impugnazioni successive alla prima non dovesse essere formulata in via incidentale, ma potesse essere articolata liberamente, anche in processi autonomi, si introdurrebbe un elemento distonico rispetto al sistema delineato dal legislatore. Nel sistema si aprirebbe una breccia dalla quale troverebbero facile accesso marcate ed evidenti elusioni del principio di concentrazione delle impugnazioni.

In definitiva, quanto precede induce a ritenere che l'impugnazione successiva alla prima va formulata in via incidentale a pena di decadenza. Pertanto, va condivisa la tesi secondo la quale a seguito dell'emanazione della predetta disciplina, non può essere condiviso il pregresso orientamento giurisprudenziale secondo il quale la riunione delle impugnazioni è idonea a evitare la decadenza delle stesse ${ }^{45}$. Invero, la decadenza si verifica già al momento della proposizione della impugnazione, indipendentemente dal momento in cui venga dichiarata. La riunione delle impugnazioni, invece, interviene sempre dopo la proposizione delle stesse e, quindi, successivamente al verificarsi della decadenza. In assenza di una espressa disposizione che conferisca alla decisione del giudice di riunire i processi una funzione sanante della decadenza, la tesi della giurisprudenza non può essere accolta ${ }^{46}$.

La tesi giurisprudenziale, secondo la quale la riunione disposta dal Consiglio di Stato evita la declaratoria di decadenza, non può

44 S. PERONGINI, Le impugnazioni nel processo amministrativo, Milano, 2011, 88 ss

45 S. PERONGINI, Le impugnazioni nel processo amministrativo, Milano, 2011, 86.

46 In tal senso, M.A. MERCATI, Commento all'art. 96 c.p.a., in R. GAROFOLI, G. FERRARI (a cura di), Codice del processo amministrativo, Roma, 2010, 1378; S. PERONGINI, Le impugnazioni nel processo amministrativo, Milano, 2011, 85 ss. 
essere accolta perché lascia arbitro il giudice di decidere quando determinare la decadenza dell'impugnazione. Il giudice, decidendo quando disporre la riunione delle impugnazioni, condiziona la sorte delle impugnazioni proposte autonomamente. La mancata riunione causa la decadenza di queste ultime.

Il codice del processo amministrativo, richiamando l'art. 333 c.p.c., sostituisce a una valutazione del giudice da compiersi caso per caso, una valutazione compiuta una volta per tutte, in termini astratti e generali dal legislatore.

La decadenza, proprio perché diretta ad assicurare l'attuazione del principio di concentrazione delle impugnazioni e delle finalità alle quali questo è preposto, non può essere lasciata all'arbitrio del giudice o al caso, ma va prevista come sanzione certa e oggettiva, connessa a fattispecie tipiche e tassative.

Una siffatta eventualità non è preclusa dal fatto che l'art. 96, primo comma, c.p.a. sancisce che «tutte le impugnazioni proposte separatamente contro la stessa sentenza devono essere riunite in un solo processo». La preclusione della predetta eventualità non deriverebbe dal fatto che il legislatore abbia previsto la riunione come l'esito di un potere discrezionale esercitato dal giudice, bensì come una conseguenza necessaria della proposizione di più impugnazioni proposte contro la stessa sentenza.

Invero, anche se dalla succitata norma emerge che la riunione delle impugnazioni costituisce un dovere per il giudice, ciò non toglie che possano verificarsi casi in cui la riunione non venga disposta.

Le ragioni per le quali il giudice può omettere di riunire le impugnazioni sono le più diverse e non sono rilevanti ai fini che qui direttamente interessano. L'eventualità che non venga disposta la riunione di più impugnazioni proposte contro la stessa sentenza è contemplata dallo stesso codice del processo amministrativo. Invero, lo stesso art. 96, sesto comma, c.p.a., fa riferimento ad una ipotesi in cui sia sta omessa la riunione di più impugnazioni, stabilendo le conseguenze che derivano dal fatto che sia stata decisa una delle impugnazioni. L'art. 96, sesto comma, c.p.a. sancisce che " in caso di mancata riunione di più impugnazioni ritualmente proposte contro la stessa sentenza, la decisione di una delle impugnazioni non determina l'improcedibilità delle altre».

VI. L'indagine sino ad ora condotta impone di verificare quali connotazioni possegga l'impugnazione incidentale tardiva, in che 
termini sia proponibile e se il riferimento, contenuto nel codice del processo amministrativo, alla categoria delle impugnazioni incidentali tempestive e a quelle tardive abbia condotto alla scomparsa della figura delle impugnazioni incidentali così dette proprie.

L'art. 96, quarto comma, c.p.a., con una disposizione innovativa rispetto ai pregressi orientamenti giurisprudenziali, introduce la figura dell'impugnazione incidentale tardiva ${ }^{47}$.

Le ragioni per le quali il nostro legislatore introdusse nel processo civile la figura delle impugnazioni incidentali tardive sono note ${ }^{48}$.

Spesso, coloro che erano usciti parzialmente soccombenti da una controversia reputavano preferibile accontentarsi del risultato raggiunto ed evitare di proporre impugnazione. Di fronte al rischio di veder vanificato l'esito conseguito, i soccombenti parziali preferivano accontentarsi della soluzione data dal giudice alla lite. Tuttavia, il pericolo che la controparte, a sua volta soccombente parziale, potesse impugnare, magari l'ultimo giorno utile, induceva anche coloro che, malgrado tutto, si sentivano soddisfatti dalla soluzione offerta al caso controverso, a proporre impugnazione a scopo prudenziale ${ }^{49}$.

Le impugnazioni proposte per fini precauzionali erano volte ad evitare che l'impugnazione potesse svolgersi in malam partem, come conseguenza del fatto che il giudice fosse stato chiamato a pronunciarsi solo sulle censure prospettate nel gravame articolato da controparte.

47 Sull'impugnazione incidentale tardiva, G. PALEOLOGO, L'appello, 1989, 571 ss., 575 ss.; V. CAIANIELLO, Manuale, 2003, 930 ss.; G. LEONE, Il sistema, 2006, 307 ss.; G. CARLOTTI, L'appello incidentale, l'appello parziale, la riserva di appello e l'appello contro il dispositivo, 2008, 539 ss; S. VALAGUZZA, Le nuove norme in tema di impugnazioni incidentali e la disciplina della traslatio a partire dai chiarimenti resi dall'Adunanza Plenaria n. 24/2011, in Dir. proc. amm., 2013, 271 ss.

48 Sull'impugnazione incidentale tardiva nel processo civile, S. CHIARLONI, L'impugnazione incidentale nel processo civile, Milano, 1969, 285 ss.; A. CERINO CANOVA, Impugnazione incidentale tardiva su oggetto diverso da quello dell'impugnazione principale, in Giur. it., 1973, I, 2, 1233 ss.; A. CERINO CANOVA, Una specie in via di estinzione: l'impugnazione incidentale tardiva, in Giur. it., 1975, I, 1, 855 ss.; A. CERINO CANOVA, Impugnazioni separate contro la stessa sentenza, in Riv. dir. proc., 1976, 298 ss.; A. CERINO CANOVA, Frammenti di novità riguardo all'impugnazione incidentale tardiva, in Giur. it., 1983, I, 1, 1 ss.; A. BONSIGNORI, Impugnazioni civili in genere, in Dig. disc. priv., sez. priv., IX, 1993, 349 ss.; C. CONSOLO, Il processo di primo grado e le impugnazioni delle sentenze, Padova, 2009, 393 ss.; C. MANDRIOLI, Corso di diritto processuale civile, II, Torino, 2010, 262 ss.

49 Sulla ratio delle impugnazioni incidentali tardive, si vedano S. PERONGINI, Le impugnazioni, op. cit., 87; S. VALAGUZZA, Le nuove norme in tema di impugnazioni incidentali e la disciplina della traslatio a partire dai chiarimenti resi dall'Adunanza Plenaria n. 24/2011, in Dir. proc. amm., 2013, 277 ss. 
Per evitare tutto ciò, anche coloro che si sentivano soddisfatti del parziale risultato raggiunto o che comunque preferivano evitare il rischio di veder messo di nuovo tutto in discussione erano costretti a proporre impugnazione. Gli uffici giudiziari erano pieni di gravami, non sempre retti da un reale interesse a coltivare l'impugnazione, i quali, però, inesorabilmente intralciavano e ritardavano lo svolgimento dei processi. Il legislatore ritenne di intervenire al fine di evitare o ridurre il fenomeno delle impugnazioni proposte a fini meramente prudenziali o precauzionali.

Per ridimensionare il fenomeno in questione, si optò per una soluzione idonea a riequilibrare la posizione delle parti in sede di gravame. Il riequilibrio della posizione fu ritenuto possibile consentendo alle parti del processo il potere di articolare, dopo la proposizione dell'impugnazione principale, una impugnazione incidentale tardiva.

L'impugnazione incidentale tardiva venne introdotta nel codice di procedura civile al fine di evitare che le parti proponessero impugnazioni solo a scopo precauzionale. L'art. 334 c.p.c. conferì il potere di proporre impugnazioni incidentali tardive sia alle parti contro le quali era stata proposta l'impugnazione, sia alle parti nei cui confronti era stata ordinata l'integrazione del contraddittorio.

La finalità pratica dell'istituto della impugnazione incidentale tardiva è quella di evitare che le parti siano costrette a proporre impugnazioni per scopi precauzionali o prudenziali ${ }^{50}$.

La ratio sottesa all'istituto è stata oggetto di un'attenta ricerca. Si è sostenuto che la ratio andasse individuata nell'esigenza di ripristinare l'equilibrio fra le parti, alterato dalla proposizione del gravame nell'ultimo periodo per impugnare ${ }^{51}$, oppure in quella di assicurare anche in fase di gravame il pieno rispetto del principio del contraddittorio $^{52}$, o infine in un duplice fondamento, soggettivo e oggettivo. Quello soggettivo era determinato dall'interesse autonomo non collegato alla proposizione dell'impugnazione principale; quello ogget-

50 Sulla disciplina dell'impugnazione incidentale tardiva, C. CONSOLO, Il processo di primo grado e le impugnazioni delle sentenze, Padova, 2009, 393 ss.; C. CONSOLO, Spiegazioni di diritto processuale civile, III, Il processo di primo grado e le impugnazioni delle sentenze, Padova, 2010, 276 ss.

51 S. COSTA, Manuale di diritto processuale civile, Torino, 1966, 441; A. ATTARDI, Limiti di applicazione del gravame incidentale tardivo, in Riv. dir. proc., 1965, 173 , 184.

52 F. CARPI, Note sui limiti di applicazione dell'impugnazione incidentale tardiva, in Riv. trim. proc. civ., 1966, 714. 
tivo, invece, era rappresentato dall'esigenza di provvedere all'unicità del giudizio ${ }^{53}$.

La giurisprudenza civile ha dato l'impressione di aver recepito con difficoltà la norma in questione. Per un lungo periodo ha dominato un orientamento che si prefiggeva di ridurre in margini angusti l'efficacia dell'innovazione legislativa. In tal senso, vanno richiamate numerose decisioni secondo le quali l'impugnazione incidentale tardiva poteva essere proposta solo avverso lo stesso capo della sentenza oggetto dell'impugnazione principale.

Inoltre, la giurisprudenza ha ristretto il campo di operatività della disciplina introdotta dall'art. 334 c.p.c. enfatizzando quello che è stato definito il limite soggettivo ${ }^{54}$.

La dottrina sottopose gli orientamenti giurisprudenziali a una serrata critica, che condusse una parte della giurisprudenza a rimeditare le proprie posizioni.

Nella giurisprudenza amministrativa il limite oggettivo, rappresentato dalla necessità che l'impugnazione incidentale tardiva avesse come oggetto lo stesso capo di sentenza censurato con l'impugnazione, attecchì favorevolmente ${ }^{55}$. Invero, i giudici amministrativi erano propensi a ridurre la portata normativa dell'art. 334 c.p.c. perché intravedevano nella norma un escamotage che avrebbe potuto condurre a una elusione della perentorietà del termine per impugnare ${ }^{56}$.

Sennonché anche nel processo amministrativo si produssero gli effetti causati dalle critiche articolate dalla dottrina processualcivilistica $^{57}$ e dalla giurisprudenza della cassazione. Una importante sentenza della Cassazione ${ }^{58}$ segnò una vera e propria inversione di tendenza rispetto all'orientamento consolidato.

53 E. GRASSO, Le impugnazioni incidentali, Milano, 1973, 33, 43 ss.

54 Sulla così detta limitazione soggettiva si vedano C. CONSOLO, Il processo, op. cit., 2009, 294 ss.; C. CONSOLO, Spiegazioni di diritto processuale civile, III, Il processo, op. cit., 2010, 277 ss.

55 In tal senso, Cons. Stato, V, 14.05.1986 n. 254, in Cons. st., 1986, I, 675; Cons. Stato, V, 18.10.1985 n. 331, in Cons. st., 1985, I, 1127; Cons. Stato, V, 18.06.1984 n. 470, in Cons. st., 1984, I, 798.

56 In tal senso, G. CARLOTTI, L'appello incidentale, op. cit., 2008, 541 ss.

57 Il riferimento più diretto è a E. GRASSO, Le impugnazioni incidentali, op. cit., 1973, 75 ss.

58 Cass., II, 28.02.1987 n. 2149 in Giur. it., 1989, I, 200 ss., con nota di Rapisarda, A piccoli passi verso il superamento dei limiti oggettivi di ammissibilità dell'impugnazione incidentale tardiva. 
La quarta sezione del Consiglio di Stato rimise alla Adunanza plenaria la questione del limito oggettivo ${ }^{59}$. L'Adunanza plenaria del Consiglio di Stato perse una importante occasione per definire la questione, perché glissò il problema, sostenendo che nel caso sottoposto al sue esame vi era connessione fra i capi di sentenza rispettivamente oggetto dell'impugnazione principale e dell'impugnazione incidentale tardiva. Secondo l'Adunanza plenaria la connessione fra i diversi capi di sentenza ricorreva sicuramente nei casi in cui i capi facevano riferimento a provvedimenti già di per sé connessi ${ }^{60}$.

Negli anni successivi la giurisprudenza ha continuato a oscillare fra la tesi dell'irrilevanza del requisito oggettivo ${ }^{61}$ e quella della identità oggettiva dei capi di sentenza impugnati con l'appello principale e con quello incidentale tardivo ${ }^{62}$.

L'art. 96, secondo e quarto comma, c.p.a. richiama proprio l'istituto previsto dall'art. 334 c.p.c. e lo rende applicabile anche nel processo amministrativo. La possibilità di proporre impugnazione incidentale tardiva sussiste anche quando si sia lasciato decorrere il termine per impugnare o si sia prestata acquiescenza alla sentenza. Anche l'impugnazione incidentale tardiva può essere proposta avverso capi autonomi della sentenza.

Il codice del processo amministrativo ha previsto un rapporto di condizionalità dell'impugnazione incidentale tardiva con l'impugnazione principale. Invero, l'art. 96, quarto comma, c.p.a. stabilisce che "se l'impugnazione principale è dichiarata inammissibile, l'impugnazione incidentale perde ogni efficacia».

Tuttavia, la norma omette di pronunciarsi su altre fattispecie pure significative in cui l'impugnazione principale viene meno. A tal proposito, va annoverato il caso della rinunzia all'impugnazione principale che, pur non essendo ricompreso tra le ipotesi richiamate dalla succitata disposizione, non mancherà di sollecitare un dibattito giurisprudenziale sulla sua idoneità a determinare l'inefficacia dell'impugnazione incidentale. Su questi temi occorrerà ritornare nel paragrafo dedicato espressamente al rapporto di condizionalità.

VII. Il codice del processo amministrativo consente la proposizione dell'impugnazione incidentale tardiva anche ai destinatari della notifica dell'impugnazione, disposta su ordine del giudice di

59 Cons. Stato, IV, 23.11 .1988 n. 875, ord., in Cons. st., 1988, I, 1367.

60 In tal senso, Cons. Stato, Ad. plen., 15.03.1989 n. 5, in Foro amm., 1989, I, 493.

61 Cfr., Cons. Stato, VI, 15.19.1990 n. 916, in Cons. st., 1990, I, 1268; Cons. Stato, V, 8.04.1991 n. 458, in Cons. st., 1990, I, 667.

62 Cfr., Cons. Stato, V, 1.02.1990 n. 60, in Cons. st., 1990, I, 208. 
integrare il contraddittorio. La possibilità di proporre impugnazioni incidentali conseguenti all'integrazione del contraddittorio pone il problema di capire se il principio di concentrazione del contraddittorio è attuato in maniera integrale e completa.

Si è sostenuto che il codice del processo amministrativo non attua in maniera piena il principio di concentrazione delle impugnazioni nel processo amministrativo. Secondo il predetto orientamento, poiché nelle "causa inscindibile e cause dipendenti» l'impugnazione deve essere notificata "a tutte le parti in causa», mentre, nei c.d. "altri casi», l'impugnazione va notificata «alle parti che hanno interesse a contraddire», l'area delle parti tenute a proporre le proprie impugnazioni in via incidentale è più ristretta rispetto a quella stessa area individuabile nel processo civile ${ }^{63}$.

Invece, il codice di procedura civile assicura la concentrazione delle impugnazioni in maniera più intensa di quanto non faccia il codice del processo amministrativo. Infatti, poiché l'art. 332 c.p.c. stabilisce che in sede di integrazione del contraddittorio la notifica dell'impugnazione vada fatta a tutte le parti, nei cui confronti l'impugnazione non sia preclusa o esclusa, l'area dei soggetti tenuti a proporre la loro impugnazione in via incidentale è tanto estesa quante sono la parti del processo che non siano già decadute dal potere di proporre impugnazione, che non abbiano fatto acquiescenza o non abbiano rinunciato alla impugnazione ${ }^{64}$.

Invece, il codice del processo amministrativo delimita un'area decisamente più ristretta dei soggetti che sono tenuti alla proposizione dell'impugnazione in via incidentale ${ }^{65}$.

Infatti, l'art. 333 c.p.c. prevede che i soggetti che devono proporre impugnazione incidentale sono tutte le parti destinatarie della notifica, sia nelle ipotesi oggetto dell'art. 331, che in quelle oggetto dell'art. 332 c.p.c. ${ }^{66}$

Sennonché, le parti destinatarie della notifica dell'atto di impugnazione, ai sensi dell'art. 95, primo comma, c.p.a., sono nelle «causa inscindibile e cause dipendenti» tutte le parti in causa; mentre, negli «altri casi», sono soltanto le parti che «hanno interesse a contraddire». Invero, l'art. 95, primo comma, c.p.a. sancisce che negli "altri casi», vale a dire nelle cause scindibili, l'integrazione debba investire non tutte le parti del processo, ma solo quelle «interessate

63 S. PERONGINI, Le impugnazioni, 2011, 77.

64 S. PERONGINI, Le impugnazioni, 2011, 77.

65 S. PERONGINI, Le impugnazioni, 2011, 77.

66 S. PERONGINI, Le impugnazioni, 2011, 77. 
a contraddire». In tal modo, l'area dei soggetti tenuti a proporre la propria impugnazione in via incidentale comprende non solo tutti coloro che sono titolari di una posizione contrastante con l'impugnante principale, ma anche coloro che, pur avendo una posizione autonoma, si collocano in una posizione di cointeresse con l'impugnante principale ${ }^{67}$.

Pertanto, «negli altri casi», i soggetti che sono tenuti alla proposizione dell'impugnazione incidentale sono soltanto le parti che «hanno interesse a contraddire».

In definitiva, nel processo amministrativo, è più elevato, rispetto al processo civile, il rischio che nei confronti della medesima sentenza, siano proposte più impugnazioni separate ${ }^{68}$.

VIII. Altro problema da affrontare è quello volto a verificare quale rapporto intercorra tra l'impugnazione principale e l'impugnazione incidentale.

Per offrire al tema innanzi prospettato una soluzione appagante occorre abbandonare la tralaticia convinzione secondo la quale si sia in presenza di un problema che richieda uno svolgimento e una soluzione unitaria. L'assioma cha ha dominato per decenni le trattazioni dell'argomento consisteva nella constatazione che l'impugnazione incidentale riceveva linfa vitale solo dal ricorso incidentale, il quale, nel contempo, costituiva anche il modello paradigmatico di riferimento.

Invero, la derivazione della disciplina dell'impugnazione incidentale, in virtù dell'art. 29 l.n. 1034/1971, dall'art. 22 t. u. 1058/1924 e dall'art. 37 t. u. 1054/1924, dettati esclusivamente per il ricorso incidentale innanzi al Consiglio di Stato, aveva causato che l'unica figura di impugnazione era l'appello incidentale ${ }^{69}$ e che il suo parametro di riferimento era costituito dal ricorso incidentale in primo grado. L'unica impugnazione era rappresentata dall'impugnazione incidentale propria, vale a dire quella fondata da una soccombenza formale o teorica.

\footnotetext{
67 S. PERONGINI, Le impugnazioni, 2011, 77.

68 In tal senso, A. TRAVI, Lezioni, IX, 2010, 309; S. PERONGINI, Le impugnazioni, 2011, 77.

69 Sull'appello incidentale si veda Cons. Stato, Ad. plen., 22.12.1982 n. 21, con nota di F. LUBRANO, Osservazioni in tema di appello incidentale, in Dir. proc. amm., 1984, 237 ss.; Cons. Stato, VI, 25.02.1989 n. 173, con nota di D. ARDINI, Ancora un tentativo di unificazione delle opposte tesi in materia di appello incidentale, in Dir. proc. amm., 1989, 453 ss.
} 
Come è noto, l'impugnazione incidentale propria può essere proposta dal soccombente in senso formale. Invero, si ha impugnazione incidentale propria quando l'interesse a impugnare non sgorga dalla sentenza, bensì dalla proposizione dell'impugnazione principale.

La sua caratteristica risiede nel rapporto di condizionalità-dipendenza con l'impugnazione principale.

Tuttavia, l'innesto delle norme del codice di procedura civile nel sistema processuale amministrativo sulle impugnazioni incidentali ben presto rivelò l'iniquità dell'applicazione del rapporto di accessorietà e subordinazione, contemplato dall'art. 37 , sesto comma, t. u. 1054/1924, a tutte le impugnazioni incidentali. Infatti, la rinunzia o la declaratoria di inammissibilità dell'impugnazione principale travolgeva tutte le impugnazioni proposte in via incidentale, anche se supportate da un autonomo interesse a impugnare.

Da ciò sorse l'esigenza di distinguere fra appello incidentale proprio e appello incidentale improprio ${ }^{70}$.

L'appello incidentale proprio $^{71}$ era quello proponibile solo dal soccombente formale, vale a dire dalla parte processuale che, pur avendo visto respinte alcune sue eccezioni, era risultata vincitrice del processo. Quest'ultima circostanza precludeva al soccombente la possibilità di impugnare la sentenza, che pur aveva respinto alcune sue eccezioni, perché priva dell'interesse a impugnare. La proposizione dell'impugnazione da parte del soccombente sostanziale determinava il sorgere dell'interesse a impugnare del soccombente formale e gli consentiva la proposizione dell'impugnazione incidentale. L'esistenza di un rapporto di accessorietà e di subordinazione fra l'appello incidentale proprio e l'appello principale giustificava l'applicazione dell'art. 37, sesto comma, t. u. 1054/1924.

L'appello incidentale improprio ${ }^{72}$, invece, era proposto da un soggetto in posizione di soccombenza sostanziale e ricorreva ogni qual volta una impugnazione sostanzialmente autonoma veniva esperita in via incidentale solo perché formulata successivamente alla notifica di altra impugnazione, che assumeva la denominazione di appello principale. In questi casi, l'appellante incidentale improprio era sollecitato da un autonomo interesse a impugnare, non accessorio,

70 Sulla distinzione fra appello incidentale proprio e appello incidentale improprio cfr. V. CAIANIELLO, Manuale, 2003, 923 ss.; G. LEONE, Il sistema, 2006, 282 ss.; N. SAITTA, Sistema di giustizia amministrativa, Milano, 2012, 781 ss.

71 S. PERONGINI, Le impugnazioni, 2011, 86 ss.

72 S. PERONGINI, Le impugnazioni, 2011, 80 ss. 
né subordinato a quello dell'appellante principale. In questi casi, l'art. 37, sesto comma, t. u. 1054/1924 non poteva essere applicato.

In definitiva, prima dell'emanazione del codice del processo amministrativo, le impugnazioni incidentali erano pacificamente ammesse nel processo amministrativo, anche se la carenza di una specifica normativa rendeva problematico l'assetto di diversi profili ${ }^{73}$. In particolare, si discuteva se l'omessa proposizione dell'impugnazione in via incidentale ne determinasse la decadenza, se l'impugnazione incidentale fosse proponibile solo avverso lo stesso capo di sentenza, se la rigorosa disciplina prevista dall'art. 37, sesto comma, t. u. 1054/1924 trovasse applicazione anche nei confronti di impugnazioni sostenute da un autonomo interesse a impugnare, ecc. ecc.

Tuttavia, a seguito dell'emanazione dell'art. 96 c.p.a. diventa problematico stabilire se l'impugnazione incidentale propria conservi una sua autonomia disciplinare. Infatti, l'art. 96 c.p.a. detta un'unica disciplina applicabile sia alle ipotesi di impugnazione incidentale propria, che ai casi di impugnazione impropria.

L'impugnazione incidentale propria, analogamente a quella impropria, può essere proposta, diversamente da quanto si riteneva in passato, anche avverso altri capi autonomi della sentenza, e i termini per la notificazione e per il deposito dell'impugnazione incidentale propria sono gli stessi di quelli previsti per l'impugnazione incidentale impropria.

Alla coppia «impugnazione incidentale propria - impugnazione incidentale impropria» viene sostituita quella basata sulla distinzione «impugnazione tempestiva e impugnazione tardiva».

Il rapporto di condizionalità-dipendenza della impugnazione incidentale propria dall'impugnazione principale non è più oggetto di una specifica disciplina. L'abrogazione dell'art. 37 t.u. 1054/1924, ad opera dell'art. 4, primo comma, n. 4, allegato 3 al Codice, non comporta più automaticamente l'inefficacia dell'impugnazione incidentale, qualora quest'ultima venga prodotta dopo che si sia rinunziato all'impugnazione principale, o qualora quest'ultima venga dichiarata inammissibile, per essere stata proposta fuori termine.

73 Sottolinea le diverse posizioni della giurisprudenza R. VILLATA, Incertezza in tema di appello incidentale nel processo amministrativo, in Dir. proc. amm., 1984, 159 ss.; R. VILLATA, Ancora in tema di appello incidentale, in Dir. proc. amm., 1986, 155 ss.; R. VILLATA, L'appello incidentale innanzi all'Adunanza Plenaria, in Dir. proc. amm., 1989, 316 ss.; R. VILLATA L'Adunanza Plenaria perde un'occasione per chiarire i problemi dell'appello incidentale, ma poi (forse) ripara, in Dir. proc. amm., 1989, 747 ss. 
Tutto ciò rende estremamente problematica la persistenza di un rapporto di condizionalità fra l'impugnazione principale e l'impugnazione incidentale, soprattutto quella così detta impropria, e potrebbe indurre a ritenere ormai superata la distinzione in questione.

Invece, il rapporto di condizionalità-dipendenza della impugnazione incidentale da quella principale ricorre fra impugnazione incidentale tardiva e impugnazione principale. Invero, ai sensi dell'art. 96, quarto comma, c.p.a. e dell'art. 334, secondo comma, c.p.c., se l'impugnazione principale è dichiarata inammissibile, l'impugnazione incidentale tardiva perde ogni efficacia.

La nuova disciplina pone la necessità di affrontare il problema di verificare se nelle due coppie di impugnazioni (impugnazione incidentale propria e impropria, impugnazione incidentale tempestiva e tardiva) permanga un rapporto di condizionalità-dipendenza e quali caratteristiche esso possegga. In particolare, occorrerà acclarare nelle due coppie quale sia il fondamento normativo del rapporto di condizionalità-dipendenza, quali siano i fondamenti logici e teorici, quali siano le conseguenze, se il venir meno dell'impugnazione incidentale conduca alla stessa o a distinte declaratorie di estinzione del processo e, infine, quale natura esso abbia.

Innanzi tutto, va evidenziato che il fondamento normativo del rapporto di condizionalità-dipendenza è diverso nelle due coppie di tipologie di impugnazioni. Il rapporto fra impugnazione incidentale principale e impugnazione incidentale propria era disciplinato dall'art. 37 t.u. 1054/1924. La norma, come è noto, è stata abrogata dal codice del processo amministrativo e il legislatore non l'ha sostituita con un'altra, per cui il predetto rapporto non è oggetto di alcuna disciplina specifica. Invece, il rapporto fra impugnazione incidentale tempestiva e impugnazione incidentale tardiva è disciplinato espressamente dall'art. 94, quarto comma, c.p.a.. le considerazioni che verranno sviluppate successivamente consentiranno di acclarare quale sia il fondamento normativo del rapporto di condizionalitàdipendenza nella coppia di impugnazioni principale e incidentale propria. Pertanto, al momento è sufficiente rimarcare che le due coppie di impugnazione non sono soggette allo stesso trattamento normativo.

L'indagine, inoltre, conduce alla scoperta che fra le due coppie di rapporti sussiste una profonda diversità dei reciproci fondamenti logici e teorici. 
L'abrogazione dell'art. 37 t.u. 1054/1924 non determina il venir meno del rapporto di condizionalità-dipendenza fra impugnazione principale e impugnazione incidentale propria, ma determina il venir meno del rapporto così come era disciplinato dalla predetta norma e come era ritenuto dalla giurisprudenza. Come si vedrà, esso assumerà un fondamento logico e teorico diverso.

Innanzi tutto, il rapporto di condizionalità ricorrerà nell'ipotesi di soccombenza formale.

Invero, l'abrogazione normativa non ha comportato il venir meno della distinzione fra impugnazione incidentale propria e impugnazione incidentale impropria. La predetta distinzione non potrà trovare la sua ragione genetica nell'art. 37 t.u. 1054/1924, ma assumerà una diversa dislocazione teorica. La distinzione si basa sulla sussistenza dell'interesse a impugnare.

Invero, in applicazione dei principi generali in tema di interesse a impugnare, qualora per qualsiasi causa venga meno l'impugnazione principale, si verificherà automaticamente l'inammissibilità dell'impugnazione incidentale propria per sopravvenuto difetto di interesse. Come si è innanzi evidenziato, l'interesse a impugnare è una condizione dell'azione. Pertanto, la sua presenza è necessaria non solo nella fase incoativa del processo, ma anche durante tutto il suo svolgimento fino all'emanazione della sentenza ${ }^{74}$.

Invece, quando l'interesse a impugnare è autonomo, vale a dire quando la sentenza determina una soccombenza sostanziale, si sarà in presenza di una impugnazione incidentale impropria. In questo caso, le sorti dell'impugnazione principale non condizioneranno l'impugnazione incidentale, che sopravviverà al venir meno della prima.

Invece, quando l'interesse a impugnare non è autonomo, vale a dire quando la sentenza determina una soccombenza formale, si sarà in presenza di una impugnazione incidentale propria. In questo caso, le sorti dell'impugnazione principale condizioneranno l'impugnazione incidentale, che sarà travolta con il venir meno della prima. In quest'ultima ipotesi, l'impugnazione incidentale propria sarà travolta da una declaratoria di inammissibilità per carenza di interesse a impugnare, qualora per una qualsiasi ragione venga meno l'impugnazione principale. In detta ipotesi il rapporto di condizionalità è destinato a permanere, ma assumerà una diversa giustificazione.

74 Sembra di contrario avviso il Cons. Stato, Ad. plen., 16.12.2011 n. 24, la quale afferma in generale che «l'impugnazione incidentale di cui all'art. 333 c.p.c. non è condizionata all'esito di quella principale, nel senso che resta efficace anche se quella principale è dichiarata inammissibile». 
Inoltre, il rapporto di condizionalità-dipendenza ricorre nella coppia impugnazione tempestiva e impugnazione incidentale tardiva. I fondamenti logici e teorici, in questo caso, andranno ravvisati nell'esigenza di evitare che attraverso un'attenta regia si eluda sistematicamente il termine perentorio concesso dal legislatore per proporre l'impugnazione.

Pertanto, fra le due coppie di impugnazioni, il rapporto di condizionalità-dipendenza possiede diversi fondamenti logici e teorici.

Le considerazioni che precedono consentono di precisare un aspetto prima necessariamente lasciato in sospeso. Invero, mentre il rapporto di condizionalità-dipendenza nella coppia impugnazione tempestiva e impugnazione incidentale tardiva ha un fondamento normativo diretto nell'art. 96, quarto comma, c.p.a.; il rapporto di condizionalità-dipendenza nella coppia impugnazione principale e impugnazione incidentale propria ha un fondamento normativo indiretto nell'art. 39, primo comma, c.p.a. e nell'art. 100 c.p.c. che disciplina, come è noto, l'interesse ad agire.

Anche le conseguenze del rapporto di condizionalità-dipendenza sono differenti nelle diverse coppie di impugnazioni. Per la coppia «impugnazione principale - impugnazione incidentale tardiva», l'art. 96, quarto comma, c.p.a. stabilisce che «se l'impugnazione principale è dichiarata inammissibile, l'impugnazione incidentale perde ogni efficacia».

La norma pone tre problemi: un primo problema di qualificazione delle conseguenze connesse alla declaratoria di inammissibilità dell'impugnazione principale, un secondo problema volto a verificare se il venir meno dell'efficacia dell'impugnazione incidentale tardiva sia connesso solo all'ipotesi dell'impugnazione di un capo autonomo della sentenza e un terzo problema consistente nel verificare quali siano le conseguenze derivanti dalle altre cause di estinzione dell'impugnazione incidentale.

a) Il primo problema di qualificazione giuridica meriterebbe una attenta esegesi e una complessa trattazione, che in questa sede non è possibile svolgere per esigenze di economia dell'indagine. La questione può essere definita prospettando solo una possibile soluzione. Invero, la locuzione "perde ogni efficacia» non configura un'autonoma categoria teorica, ma rappresenta un termine atecnico, il cui significato va ricostruito alla luce della classificazione introdotta dall'art. 35 c.p.a. fra le diverse tipologie di pronunce di rito. 
b) Il secondo problema verrà affrontato successivamente nel paragrafo dedicato alla questione della censurabilità dello stesso capo o anche di capo autonomo di sentenza.

c) Il rapporto di condizionalità-dipendenza dell'impugnazione incidentale tardiva all'impugnazione principale deriva dalla finalità attribuita all'istituto di assicurare una condizione di parità delle armi riservata alle parti nella fase delle impugnazioni, consentendo alla parte che si sia accontentata degli esiti della pronuncia sul presupposto dell'inerzia della controparte, dettata da analogo atteggiamento di accettazione, di proporre a sua volta impugnazione, anche tardiva, qualora la condizione di equilibrio sia stata alterata dall'impugnazione di una delle parti.

Con il venir meno, per una ragione qualsiasi dell'impugnazione principale, viene meno anche la ragione di consentire all'altro soccombente di far valere le sue pretese impugnando tardivamente la sentenza.

Inoltre, occorre verificare quale sia la declaratoria nel caso in cui venga meno l'impugnazione principale.

Nella coppia impugnazione principale e impugnazione incidentale tardiva, l'inammissibilità dell'impugnazione principale, ai sensi dell'art. 35 c.p.a., costituisce una ragione sopravvenuta, ostativa alla pronuncia sul merito, causa di improcedibilità del ricorso.

Anche nella coppia «impugnazione principale - impugnazione incidentale propria», al venir meno dell'impugnazione principale consegue l'improcedibilità dell'impugnazione incidentale propria. Il venir meno, per una qualsiasi causa, dell'impugnazione principale determina una sopravvenuta carenza di interesse dell'impugnante incidentale proprio a coltivare la sua azione. Invero, nell'impugnazione incidentale propria l'interesse a impugnare non deriva dalla soccombenza sostanziale, bensì dalla soccombenza formale o teorica, vale a dire da un interesse che sorge solo dalla proposizione dell'impugnazione incidentale. L'art. 35 c.p.a. ricollega, alla sopravvenuta carenza di interesse alla decisione, la declaratoria di improcedibilità del ricorso.

In definitiva, il veni meno dell'impugnazione principale, in tutte e due le coppie, costituisce causa di improcedibilità, ma per ragioni differenti da coppia a coppia.

Infine, rimane da affrontare la questione della natura giuridica del rapporto condizionalità-dipendenza. La presentazione dell'impugnazione principale, nelle due coppie prese in esame, condiziona 
l'articolazione dell'impugnazione incidentale. Senza la prima non è astrattamente configurabile la formulazione della seconda. Sussiste un rapporto di presupposizione. La dipendenza, invece, risiede nel fatto che il venir meno della prima causa l'improcedibilità della seconda.

IX. Occorre verificare se l'impugnazione incidentale debba essere proposta avverso il medesimo capo di sentenza, oggetto dell'impugnazione principale, oppure possa essere articolato anche avverso capi autonomi.

La nozione di capo di sentenza è stata molto dibattuta nel processo civile ${ }^{75}$, in cui sono stati prospettati tre diversi orientamenti, che hanno identificato il capo di sentenza con il "capo di domanda» ${ }^{76}$, con la soluzione di ogni questione affrontata per pervenire alla pronuncia finale ${ }^{77} \mathrm{o}$, infine, che hanno ritenuta valida la prima prospettazione per l'appello e la seconda per il ricorso per cassazione ${ }^{78}$.

\section{Il dibattito è rifluito anche nel processo amministrativo ${ }^{79}$.}

Il capo di sentenza nel processo amministrativo identifica l'entità minima della sentenza. Secondo un primo orientamento, il capo di sentenza andrebbe identificato sulla base del «petitum» del ricorso, con la conseguenza che a ogni provvedimento annullato corrisponderebbe un capo di sentenza. Un altro orientamento, invece, iden-

75 Sulla nozione di capo di domanda e su quella di capo di sentenza si vedano S. CHIARLONI, L'impugnazione incidentale nel processo civile, Milano, 1969, 29 ss.

76 Per il processo civile, come è noto, un primo orientamento, secondo il quale il «capo di sentenza» deve essere identificato con "capo di domanda» e consiste nella "unita elementare della sentenza», vale a dire in ogni frazione del suo contenuto dispositivo, è stato sostenuto da E. BETTI, Diritto processuale civile italiano, Roma, 1936, 668; P. CALAMANDREI, Appunti sulla reformatio in peius, in Riv. dir. proc. civ., 1929, I, 300; G. CHIOVENDA, Principii di diritto processuale civile, Napoli, 1960 (ma 1923), 992; A. ATTARDI, La revocazione, Padova, 1959, 103 ss.; E. FAZZALARI, Il giudizio civile di cassazione, Milano, 1960, 145 ss.; S. SATTA, Commentario al codice di procedura civile, Milano, 1966, II, 22, 53.

77 Secondo una tesi parte o capo di sentenza vanno identificati con la soluzione di ogni questione affrontata per giungere alla pronuncia finale, con la conseguenza che il parametro di riferimento non è offerto dal dispositivo, bensì dalla motivazione della sentenza. Il predetto orientamento è stato sostenuto da F. CARNELUTTI, Sulla reformatio in peius, in Riv. dir. proc. civ., 1927, 181 ss.; R. PROVINCIALI, Il giudizio di rinvio, Padova, 1936, 135 ss.; G.A. MICHELI, Corso di diritto processuale civile, Milano, 1959, II, 254 ss.

78 In tal senso, V. ANDRIOLI, Commento al codice di procedura civile, Napoli, 1956, II, 384; E. ALLORIO, Gravame incidentale della parte totalmente vittoriosa?, in Giur. it., 1956, I, 1, 543; M. NIGRO, L'appello nel processo amministrativo, Milano, 1960, 426 ss.

79 V. CAIANIELLO, Manuale di diritto processuale amministrativo, Torino, 2003, 914 ss.

(C) UNED. Revista de Derecho UNED, núm. 12, 2013 
tifica il «capo» con ogni profilo di illegittimità fatto valere. In altri termini, a ciascun vizio corrisponde un capo di sentenza. Infine, si sostiene che l'unità minima della sentenza vada identificata in ogni questione sollevata dalle parti o rilevabile di ufficio nel giudizio di primo grado. Capo di sentenza indica ogni pronuncia su un singolo vizio o su ogni singola eccezione pregiudiziale o preliminare ${ }^{80}$.

La rilevanza del dibattito nel processo amministrativo deriva dal fatto che, prima dell'emanazione del codice del processo amministrativo, l'appello incidentale proprio poteva essere proposto solo contro lo stesso capo di sentenza, gravato con l'impugnazione principale $^{81}$.

Infatti, la giurisprudenza del Consiglio di Stato, originariamente, aveva accolto una nozione ristretta di incidentalità, ritenendo che l'appello incidentale fosse ipotizzabile soltanto nel caso in cui si rivolgeva contro lo stesso capo della sentenza gravato dall'appello principale, ovvero contro un capo connesso o dipendente. Ne conseguiva che, se la parte appellata avesse proposto impugnazione incidentale, rispetto a un capo autonomo e facendo valere un autonomo interesse, e non una impugnazione autonoma principale, avrebbe visto dichiarare il suo gravame inammissibile ${ }^{82}$.

Con il codice di procedura civile nel 1940, un orientamento della giurisprudenza ammise che la parte destinataria della notifica di un atto di impugnazione, indipendentemente dalla posizione rispetto alla parte appellante in via principale, dovesse proporre impugnazione in via incidentale. Come già evidenziato, un orientamento della dottrina processualcivilista criticò la limitazione dell'impugnazione incidentale allo stesso capo di sentenza, sostenendo che si trattava di una soluzione priva di alcun riferimento normativo ${ }^{83}$.

L'orientamento in esame venne accolto anche per il processo amministrativo.

In questo modo la nozione di incidentalità venne notevolmente ampliata anche nel processo amministrativo.

Appare opportuno evidenziare che la tesi secondo la quale l'impugnazione incidentale può essere proposta solo contro lo stesso capo di sentenza avverso il quale è stata proposta l'impugnazione

80 In tal senso, A. TRAVI, Lezioni, IX, 2010, 312 ss.

81 Sul tema si veda S. PERONGINI, Le impugnazioni, op. cit., 2011, 84 ss.

82 Sul tema si veda S. PERONGINI, Le impugnazioni, op. cit., 2011, 84 ss.

83 Sul tema si veda S. PERONGINI, Le impugnazioni, op. cit., 2011, 84 ss. 
principale riduce in termini oltremodo angusti l'efficacia normativa del principio di concentrazione delle impugnazioni.

Si è evidenziato che l'art. 96, terzo comma, c.p.a., innovando rispetto al pregresso orientamento giurisprudenziale, stabilisce che "l'impugnazione incidentale di cui all'art. 333 del codice di procedura civile può essere rivolta contro qualsiasi capo di sentenza» ${ }^{84}$.

In definitiva, l'art. 96 c.p.a. afferma espressamente che l'impugnazione incidentale di cui all'art. 333 del codice di procedura civile può essere rivolta contro qualsiasi capo della sentenza.

La disposizione in parola, insieme a tutte quelle che disciplinano gli altri istituti che assicurano la concentrazione delle impugnazioni, applica in maniera rigorosa il principio di concentrazione delle impugnazioni e di esso ne costituisce conseguenza necessaria.

A seguito dell'emanazione dell'art. 96 c.p.a., il problema della definizione delle nozioni di «stesso capo o di capi autonomi di sentenza» si porrà sempre. Tuttavia, esso non avrà la ricaduta pratica assunta in passato, perché la scelta del legislatore di applicare nella maniera più ampia possibile il principio di concentrazione delle impugnazioni, imponendo forma e termini dell'impugnazione incidentale a tutte quelle proposte successivamente alla prima, esonera l'interprete dall'esegesi della norma e dalla definizione della nozione.

Appare opportuno rimarcare, in ogni caso, che la soluzione da dare al problema dell'impugnabilità dello stesso capo di sentenza o anche di capi diversi dipende esclusivamente dal grado di effettività che si voglia riservare al principio di concentrazione delle impugnazioni. È una scelta di politica giudiziaria, più che una scelta di tecnica processuale.

X. Nel percorso logico che conduce alla definizione dei profili problematici dalle impugnazioni incidentali, un passaggio fondamentale è rappresentato dalla determinazione dell'ordine di esame delle diverse impugnazione proposte.

In via preliminare, vanno formulate tre considerazioni di ordine generale.

Il tema non è oggetto di alcuna disposizione specifica, né formulata in modo espresso, né desumibile in maniera implicita dalle norme dettate in tema di impugnazione in generale. Inoltre, la questione è solo parzialmente assimilabile all'analogo problema prospettato per stabilire l'ordine di esame delle censure formulate con il ricorso

84 In tal senso si veda S. PERONGINI, Le impugnazioni, op. cit., 2011, 84 ss. 
principale e con il ricorso incidentale. Invero, il rapporto esistente fra ricorso principale e ricorso incidentale è diverso da quello corrente fra impugnazione principale e impugnazione incidentale, perché nelle impugnazioni il rapporto fra le parti è mediato dalla emanazione della sentenza di primo grado che le disloca diversamente, collocando, le une, nel novero delle parti soccombenti e, le altre, in quelle delle parti vincitrici. La soccombenza ha natura diversa dalla lesione di una situazione giuridica soggettiva di interesse legittimo.

Infine, le impugnazioni presentano una pluralità di situazioni differenti da quelle che possono verificarsi per i ricorrenti in primo grado. In primo grado, al ricorrente principale può contrapporsi il ricorrente incidentale, con la conseguente configurazione di una sola relazione. Invece, all'impugnante principale si può contrapporre l'impugnante tempestivo, l'impugnante incidentale tardivo, l'impugnante incidentale proprio e l'impugnante incidentale improprio. Ne deriva l'astratta configurabilità di ben quattro relazioni, ognuna diversa dalle altre.

L'ordine di esame delle impugnazioni, secondo un impostazione risalente, va desunto dai principi del così detto «ordine logico della pregiudizialità» in forza del quale debbono essere decise prima le questioni attinenti al processo e, poi, quelle concernenti il merito ${ }^{85}$. Il principio in parola possiede un fondamento logico, che trova le sue radici nel potere delle parti di imporre e nel dovere del giudice di compiere la verifica della sussistenza di tutti i presupposti e di tutti i requisiti desumibili dalla progressione dell'iter processuale, per giungere validamente a una pronuncia sul merito della controversia. Oggetto di verifica saranno, secondo quando previsto dall'art. 35 c.p.a., i presupposti e i requisiti di ricevibilità, ammissibilità e procedibilità.

Tuttavia, il principio in questione rinviene anche un solido fondamento nell'art. 276, secondo comma, c.p.c. secondo il quale «il collegio, sotto la direzione del presidente, decide gradatamente le questioni pregiudiziali proposte dalle parti o rilevabili di ufficio e quindi il merito della causa». Come è noto la giurisprudenza ha ripetutamente approfondito la pregiudizialità logica, offrendo un contributo di grande interesse. In particolare, costituisce un approfondimento della tematica in esame la distinzione fra le questioni pregiudiziali in senso logico e questioni pregiudiziali in senso tecni-

85 Sul tema, in dottrina L. BIANCHI D'ESPINOSA, Impugnazioni incidentali condizionate su questioni pregiudiziali, in Giust. civ., 1961, I, 1387; E. FAZZALARI, Sui ricorsi incidentali "condizionati» in Riv. trim. dir. proc. civ., 1961, 114 ss.; S. SATTA, Diritto processuale civile, Padova, 2000, 411; S. CHIARLONI, L'impugnazione incidentale nel processo civile, Milano, 1969, 124 ss. 
co. Questioni pregiudiziali in senso logico sono relative a circostanza che non rientrano nel fatto costitutivo del diritto dedotto in causa, le quali devono essere necessariamente decise incidenter tantum. Invece, le questioni pregiudiziali in senso tecnico sono quelle che rientrano nel fatto costitutivo della situazione giuridica soggettiva dedotta in causa.

L'ordine logico delle questioni da esaminare viene spesso presentato come un assioma che ha ricevuto una sistemazione definitiva dal legislatore e dall'opera ermeneutica della giurisprudenza e che, pertanto, non è suscettibile di alcuna alterazione.

L'assioma in questione è stato sottoposta a una verifica critica soprattutto dalla dottrina processualcivilistica che ha studiato le impugnazioni incidentali ${ }^{86}$. Invero, soprattutto nella fase delle impugnazioni numerosi fattori concorrono a influenzare e a condizionare l'ordine logico di esame delle questioni.

Occorre verificare se l'ordine logico delle questioni possa essere alterato dalle richieste della parte che subordini o condizioni l'esame di una questione alla verifica della fondatezza o infondatezza di altra questione.

Inoltre, occorre verificare se l'ordine logico delle questioni possa subire un'alterazione a causa delle caratteristiche quantitative e qualitative della soccombenza. Il primo problema da affrontare è quello volto a stabilire se la parte possa subordinare l'esame di una questione a un'altra, alterando l'ordine logico e giuridico dell'esame da parte del giudice.

Il secondo problema, invece, è quello volto a individuare gli effetti che il diverso dislocarsi delle parti rispetto alla sentenza provoca nell'ordine logico di esame delle questioni. In altri termini, si tratta di precisare quali effetti producano le differenti ipotesi di soccombenza sul tema in esame.

Invero, rispetto alla sentenza le parti del processo possono assumere una collocazione differente da quella che avevano originariamente. L'attore, nella fase delle impugnazioni, può assumere la veste di resistente e il convenuto, dal canto suo, può divenire a sua volta attore e viceversa.

Inoltre, a seguito dell'emanazione della sentenza in un processo con due sole parti può verificarsi che una sola parte sia soccombente sostanziale, che entrambe le parti siano in posizione di soccombenza

86 S. CHIARLONI, L'impugnazione incidentale, 1969, 126 ss. 
sostanziale reciproca, che una parte sia in posizione di soccombenza sostanziale e l'altra in posizione di soccombenza formale e teorica.

Infine, in un processo con una pluralità di parti, può verificarsi che più parti si trovino in posizione di soccombenza sostanziale, in posizione di soccombenza sostanziale reciproca o in posizione di soccombenza sostanziale e soccombenza formale o teorica.

La diversa posizione delle parti rispetto alla sentenza e la diversa natura quantitativa o qualitativa della soccombenza incidono sull'ordine di esame delle questioni, alterando lo schema logico al quale siamo abituati a pensare. In presenza di impugnazioni che consentono al giudice di conoscere questioni pregiudiziali rilevabili d'ufficio e questioni di merito, il rispetto di astratti canoni di carenza formale e di rigore giuridico imporranno prima l'esame delle questioni pregiudiziali rilevabili di ufficio e, poi, l'esame del merito. Sarebbe manifestamente illogico l'iter seguito del giudice che, prima, esami la fondatezza delle domande a lui rivolte e, poi, si soffermi sulla sussistenza di una causa preclusiva all'esame di merito, idonea a determinare la irricevibilità o improcedibilità del ricorso. In una fattispecie siffatta, il giudice si vedrà costretto a esaminare prima le questioni pregiudiziali rilevabili d'ufficio e solo all'esito di un vaglio critico positivo potrà pronunciarsi sul merito delle questioni prospettate.

In presenza, invece, di questioni pregiudiziali rilevabili esclusivamente su istanza di parte, il giudice dell'impugnazione potrà procedere al loro esame esclusivamente qualora la questione sia stata ritualmente prospettata.

Solo qualora il giudice dell'impugnazione sia investito della cognizione della questione pregiudiziale rilevabile su istanza di parte dovrà anteporre l'esame di questa a quello concernente il merito della controversia.

In ogni caso, il tema si presenta così complicato da imporre necessariamente il rinvio a successivi approfondimenti.

XI. Altro problema da porre è quello volto a stabilire se la tardività della proposizione dell'impugnazione incidentale, con conseguente declaratoria di irricevibilità o di inammissibilità, secondo $i$ casi, si debba necessariamente tradurre nell'estromissione dell'impugnante dal processo o si possa risolvere consentendogli di essere presente nel processo, ma con un ruolo minore.

Il problema va posto perché in diverse pronunce, alcune delle quali anche recenti, si è sostenuto che in caso di declaratoria di 
inammissibilità dell'impugnazione incidentale si debba convertire l'impugnazione in atto di intervento, consentendo comunque al suo autore di interloquire nel processo. In particolare, si è affermato che l'impugnazione incidentale si debba convertire in atto di intervento ad adjuvandum ${ }^{87}$. Il potere di conversione, secondo la tesi in esame, troverebbe il suo fondamento nell'art. 32 c.p.a.

La tesi non può essere accolta ${ }^{88}$.

La fattispecie disciplinata dall'art. 32 c.p.a. è quella della conversione delle azioni, cosa ben diversa dalla conversione di un atto giudiziario in un altro di cui abbia le connotazioni sostanziali.

Inoltre, non è possibile convertire una impugnazione in un atto di intervento perché fra i due atti non vi è quella identità di elementi sostanziali, che ai sensi dell'art. 32 c.p.a. costituisce il presupposto per la conversione delle azioni. L'interesse che fa valere il soggetto che propone impugnazione nasce da una situazione di soccombenza, formale o sostanziale. La soccombenza è situazione ben diversa dall'interesse che legittima la proposizione di un atto di intervento. Sono entità non commensurabili.

Peraltro, l'orientamento in esame è distonico rispetto alla tesi secondo la quale i cointeressati, che hanno omesso di presentare ricorso nei termini, non possono esplicare atto di intervento, perché diversamente si consentirebbe loro di eludere il termine perentorio per proporre il ricorso.

In ogni caso, il tema meriterebbe di essere adeguatamente approfondito. Tuttavia, in questa sede non è possibile dedicargli l'attenzione dovuta perché ciò implicherebbe un attento esame della situazione giuridica soggettiva legittimante l'atto di intervento, con il conseguente rischio di effettuare una digressione amplissima, che condurrebbe l'indagine verso altri lidi.

XII. La previsione dell'onere di proporre le impugnazioni successive alla prima in forma incidentale a pena di decadenza ricollega alla notifica dell'impugnazione principale l'effetto di modificare i termini per impugnare concessi alle altre parti ${ }^{89}$.

87 In tal senso, Cons. Stato, IV, 23.02.2012 n. 983.

88 Sul tema, S. PERONGINI, Le impugnazioni, op. cit., 2011, 76.

89 Sul tema si vedano V. ANDRIOLI, Commento, op. cit., II, 404; E. REDENTI, Diritto processuale civile, II, Milano, 1953, 335; S. CHIARLONI, L'impugnazione, op. cit., 1969, 13 ss. 
Invero, si è in presenza di uno di quei tanti casi in cui l'iniziativa processuale di una delle parti condiziona il comportamento processuale delle altre.

I termini per impugnare sono fissati perentoriamente dall'art. 92 c.p.a. in sessanta giorni - decorrenti dalla notifica della sentenza o dall'evento, all'avverarsi del quale è subordinata la proposizione della revocazione, dalla scoperta del dolo, della falsità, della collusione o dal giorno in cui è stato recuperato il documento o è passata in giudicato la sentenza - o, in mancanza della notifica, in sei mesi decorrenti dalla pubblicazione della sentenza.

Invece, in caso di proposizione dell'impugnazione principale, i suddetti termini subiscono una modifica perché l'art. 96, terzo comma, c.p.a. stabilisce che l'impugnazione incidentale tempestiva deve essere proposta dalla parte entro sessanta giorni dalla notifica della sentenza o, se anteriore, dalla notifica nei suoi confronti di altra impugnazione. Inoltre, l'art. 96, quinto comma, c.p.a., per le impugnazioni incidentali tardive, stabilisce che l'impugnazione incidentale deve essere proposta entro sessanta giorni dalla data della notifica dell'impugnazione principale.

La disciplina dettata dal codice per l'impugnazione incidentale disloca diversamente il momento a decorrere dal quale le parti, diverse dall'impugnante principale, devono articolare la loro azione. In definitiva, colui che impugna per primo, con il suo comportamento, modifica i termini entro i quali gli altri devono a loro volta proporre la propria impugnazione.

XIII. L'indagine sin qui condotta consente di fornire una risposta, per quanto approssimativa, ai problemi sopra prospettati. Le impugnazioni incidentali possono proporsi nell'appello, nella revocazione, nell'opposizione di terzo e, con qualche particolarità, anche nel ricorso per cassazione. L'impugnazione successiva alla prima «deve» (e non semplicemente "può») essere proposta in forma incidentale. L'impugnazione va proposta in forma incidentale, a pena di decadenza, dai destinatari della notifica della sentenza e dell'atto di integrazione del contraddittorio. Il codice del processo amministrativo configura diverse tipologie di impugnazioni incidentali e consente di distinguere fra impugnazioni incidentali proprie e impugnazioni incidentali improprie, nonché fra impugnazioni incidentali tempestive e impugnazioni incidentali tardive. Il rapporto di condizionalitàdipendenza, fra impugnazione principale e impugnazione incidentale, sussiste sia nel caso di impugnazione incidentale propria, sia nel caso di impugnazione incidentale tardiva. L'esigenza di applicare in 
maniera rigorosa il principio di concentrazione delle impugnazioni ha indotto il legislatore a sancire l'ammissibilità dell'impugnazione incidentale anche avverso capi autonomi della sentenza, vale a dire capi diversi da quelli oggetto dell'impugnazione principale. Tra le questioni oggetto di impugnazione principale e di impugnazione incidentale sussiste un rapporto logico e giuridico in forza del quale il loro esame deve avvenire con prevalenza delle questioni pregiudiziali su quelle di merito. Infine, si è acclarato che l'impugnazione incidentale fuori termine non può digradare ad atto di intervento. 
